THE ASTROPHYSICAL JOURNAL, 383:112-134, 1991 December 10

(C) 1991. The American Astronomical Society. All rights reserved. Printed in U.S.A.

\title{
THE ORDERED NATURE OF ELLIPTICAL GALAXIES: IMPLICATIONS FOR THEIR INTRINSIC ANGULAR MOMENTA AND SHAPES
}

\author{
MARIJN FranX \\ Harvard-Smithsonian Center for Astrophysics, 60 Garden Street, Cambridge, MA 02318 \\ GARTH ILLINGWORTH \\ Lick Observatory and University of California Observatories, University of California, Santa Cruz, CA 95064 \\ AND \\ TIM DE ZEEUW \\ California Institute of Technology; and Sterrewacht, Huygens Laboratorium, Postbus 9504, 2300 RA Leiden, The Netherlands ${ }^{1}$ \\ Received 1991 January 15; accepted 1991 June 17
}

\begin{abstract}
The observations of rotation along the major and minor axes of 38 elliptical galaxies are analyzed to determine their intrinsic structure. Rotation along the minor axis occurs (i) as a result of projection effects in triaxial systems and/or (ii) because of real misalignment of the angular momentum axis from the intrinsic short axis, as is allowed in triaxial systems. The intrinsic angular momentum can point anywhere in the plane containing the short and the long axis. The distribution of apparent misalignments is a function only of the triaxiality $T$ of a galaxy and its intrinsic misalignment, and is independent of the flattening of the galaxy in the plane containing the long and the short axis. The observed misalignment of the rotation axes and the short axes ranges from $0^{\circ}$ to $90^{\circ}$, with most ellipticals having misalignments that are small. This is not expected if ellipticals are generically triaxial systems with uniformly distributed angular momentum direction, and requires that particular constraints be imposed during the formation process. Some specific galaxies have dynamical subsystems with observed angular momenta that are offset $90^{\circ}$ from the angular momenta of the main part of the galaxy. These galaxies are probably rotating about their long axis in the outer parts. The analysis of the observed misalignments requires us to derive the intrinsic ellipticity distribution for ellipticals. The resulting distribution, based on CCD data, shows a pronounced lack of round galaxies, unlike that previously derived from catalogs. The distribution of triaxiality and intrinsic misalignment of the angular momentum cannot be derived uniquely, since one observable (apparent misalignment) is used to determine two internal parameters (triaxiality and intrinsic misalignment). Various assumptions regarding the intrinsic shapes and the direction of the angular momentum were used. An exploration of the solution space showed a wide range of valid distributions, ranging from models with only nearly oblate shapes, oblate and prolate shapes, to distributions with only triaxial shapes, with varying distributions of intrinsic misalignments. However, all the models have a sizable fraction of galaxies $(\geq 35 \%)$ with intrinsic misalignments less than $15^{\circ}$. The results were compared with predictions from several groups for hierarchical formation scenarios. The predicted distributions for halos are skewed significantly toward prolate-triaxial shapes. Our data show that the luminous parts of ellipticals do not have such high triaxialities. The shapes of the dark halos may well be different from the shapes of the luminous matter in ellipticals, despite their similar dynamics. This may result, for example, from the effect of gaseous dissipation.

Subject headings: galaxies: formation - galaxies: internal motions - galaxies: structure stars: stellar dynamics
\end{abstract}

\section{INTRODUCTION}

Ever since Binney (1978) suggested that elliptical galaxies may be triaxial, the question of their intrinsic shapes has remained unresolved. Binggeli (1980) showed that the distribution of apparent ellipticities is not sufficient to determine their shapes uniquely. Tests based on correlations between global properties such as surface brightness, velocity dispersion, and ellipticity have been inconclusive (e.g., Merritt 1982). Gas kinematics can be used to constrain the shapes of some individual galaxies (e.g., NGC 1052: Davies \& Illingworth 1986; NGC 5077: Bertola et al. 1991), but systems with readily measurable gas are too rare to allow a determination of the distribution of shapes.

\footnotetext{
${ }^{1}$ Current postal address.
}

The distribution of intrinsic shapes is relevant for a much wider range of astronomical problems than (just) the construction of equilibrium models for elliptical galaxies. Deviations from axisymmetry influence the gas kinematics in ellipticals (e.g., de Zeeuw \& Franx 1989) and may help to fuel black holes in the centers. If the intrinsic shapes of elliptical galaxies are characteristic of all collisionless systems, then galactic halos may be expected to be triaxial as well, causing observable effects in the kinematics of disks. The distribution of shapes is also of particular significance as a signature of the formation process, and could potentially be used to distinguish between different formation mechanisms.

Binney (1985) suggested a new test for the shapes of elliptical galaxies: the rotation along the apparent minor and major axis can put tight constraints on the shapes if it is assumed that the intrinsic angular momenta are well aligned with the intrinsic 
short axis. He was unable, however, to apply his test adequately because of the limited accuracy of the available data. Shortly afterward, Davies \& Birkinshaw (1986) found a galaxy rotating rapidly around its apparent long axis, confirming the prediction that elliptical galaxies can show rotation along the minor axis. More systems with rotation along the minor axis were reported by Davies \& Birkinshaw (1988), Wagner, Bender, \& Möllenhof (1988), Jedrzejewski \& Schechter (1989), and Franx, Illingworth, \& Heckman (1989b). Modern detectors have resulted in measured rotation velocities with accuracies between 5 and $10 \mathrm{~km} \mathrm{~s}^{-1}$, allowing the application of approaches such as that of Binney. Analysis of these new data to test hypotheses about the intrinsic shapes of elliptical galaxies is the central purpose of this paper.

Unfortunately, Binney's assumption that the angular momenta of galaxies are aligned with the intrinsic short axis is too limited. The apparent misalignments could in general be due to two effects. First, the projection of triaxial figures, which causes the apparent short axis to be misaligned with the projected intrinsic short axis, and, second, an intrinsic misalignment of the angular momentum with the intrinsic short axis. The second effect was not included in Binney's analysis. In general both effects will be present, and both will increase the apparent misalignment between the apparent angular momentum and the apparent short axis (although for special viewing angles they may "conspire" to reduce the apparent kinematic misalignment).

It is likely that intrinsic misalignments occur in nature. Triaxial potentials with stationary figures allow streaming about both the short axis and the long axis. These streaming motions are produced by the short and long axis tubes, respectively (e.g., Schwarzschild 1979; Binney \& Tremaine 1987). Any selfconsistent distribution function allows large freedom in the angular momentum that each individual orbit contributes to the galaxy angular momentum. Within each tube orbit, all the stars can rotate either in one sense, or in the opposite sense, or in a mixture of both. The angular momentum of the orbit can vary from zero to some maximum in either sense. Hence the angular momenta of the galaxies may point in any direction within the plane containing the short and the long axis. No further constraint can be given on purely dynamical grounds.

It is possible that the formation process is well ordered and produces close alignment between the intrinsic angular momentum and the intrinsic short axis. Various groups have found such organization to occur during simulations of hierarchical galaxy formation (e.g., Barnes \& Efstathiou 1987; Frenk et al. 1988; Quinn \& Zurek 1988; Warren et al. 1991a, b). However, all simulations produced some systems with significant misalignments between the intrinsic angular momentum and the short axis.

The galaxies with kinematically distinct cores constitute the most direct proof of the existence of systems with large intrinsic misalignments. Two Virgo systems, NGC 4365 and NGC 4406, show rotation along the minor axis in the outer parts, and no rotation along the major axis at the same radii (Bender 1988; Wagner et al. 1988; Franx et al. 1989b). In the inner few arcseconds, the galaxies have large rotation along the major axis and no rotation along the minor axis. The rotation along the minor axis in the outer parts is produced either by a projection effect of a triaxial system or by a large intrinsic misalignment. If it is caused only by projection effects, the line of sight lies in the plane containing the intrinsic long and short axes. In that case, the angular momentum of the inner parts would be caused by streaming motions around the intermediate axis. Such rotation is not allowed in triaxial galaxies, because the intermediate axis tubes are unstable (Heiligman \& Schwarzschild 1979). We therefore have to conclude that these systems have large intrinsic misalignments between the intrinsic angular momenta of the outer parts and their intrinsic short axes-probably $90^{\circ}$ (Franx et al. 1989b; Illingworth \& Franx 1990). Of course, the fact that these galaxies show twists of $90^{\circ}$ in their rotation axes is an indication by itself that the intrinsic rotation axis changes in direction; but the important point is that the outer parts are the most misaligned (and may very well rotate about their long axis)! We note in passing that kinematic twists can be produced by projection effects alone, since the shape of the streamlines can change as a function of radius. Thus, small apparent kinematic twists do not automatically imply intrinsic twists of the rotation axis. Such effects are generally too small, however, to invalidate the results for NGC 4365 and NGC 4406.

This paper is organized as follows. We analyze the kinematic misalignments under three different assumptions: First we assume that all misalignments are due to the projection effects of triaxial shapes. The intrinsic angular momentum is aligned with the intrinsic short axis for these models. Second, we assume that all misalignments are due to intrinsic misalignments. That is, the shapes are assumed to be oblate, but the angular momentum is not aligned with the intrinsic short axis. While unrealistic, such a model allows us to isolate and see the effect of intrinsic misalignment alone. Third, we construct a more general model for which the misalignments are due to both effects. The shapes are triaxial, and the angular momenta are misaligned in this case. Although our models do not cover the full solution space, they give useful upper limits on the "triaxiality" of ellipticals, and on the intrinsic misalignments. In $\S 2$ we derive the necessary equations that give the observables (ellipticities, velocities, position angles, etc.) as a function of intrinsic parameters and viewing angles. In $\S 3$ the probability distributions of the observables are derived, for specific shapes and intrinsic misalignments. In $\S 4$ we collect and discuss the data on the ellipticity distribution and misalignments. In $\S 5$ we first construct models that reproduce the apparent ellipticity distribution. We then derive three pairs of models that reproduce the histogram of apparent misalignments. The data are compared with predictions for halos from hierarchical formation scenarios in $\S 6$. The results are discussed and summarized in $\S 7$.

\section{PROJECTION OF DENSITY AND VELOCITY FIELDS}

This section summarizes the equations for the projection of the density and velocity fields.

\subsection{Projection of Density}

We assume that the density $\rho$ of a triaxial galaxy is stratified on similar coaligned ellipsoids, so that it may be written as $\rho=\rho_{m}\left(m^{2}\right)$, where $m^{2}$ is given by $m^{2}(x)=x^{2} / a^{2}+y^{2} / b^{2}+$ $z^{2} / c^{2}$. The $x$-, $y$-, and $z$-axes are symmetry axes of the density distribution. We assume that $a \geq b \geq c$, so that the $x$-axis is the long axis and the $z$-axis is the short axis of the galaxy. We define the intrinsic ellipticities $\epsilon_{1}$ and $\epsilon_{2}$ by

$$
\epsilon_{1}=1-\frac{c}{a}, \quad \epsilon_{2}=1-\frac{b}{a} .
$$

The projections of such models have been discussed by various authors, e.g., Contopoulos (1956), Stark (1977), Binney (1985), 
and Franx (1988a, hereafter F88). These authors showed that the projected density distribution is stratified on similar ellipses. The ellipticity $\epsilon$ and minor-axis position angle $\Gamma_{\text {minor }}$ of the isophotes are completely determined by the intrinsic ellipticities $\epsilon_{1}, \epsilon_{2}$ and the viewing angles. If $\phi$ and $\theta$ are the polar coordinates of the line of sight, we may write

$$
\epsilon=\epsilon\left(\epsilon_{1}, \epsilon_{2}, \phi, \theta\right), \quad \Gamma_{\text {minor }}=\Gamma_{\text {minor }}\left(\epsilon_{1}, \epsilon_{2}, \phi, \theta\right) .
$$

Franx (1988a) proved that the above relations can be simplified somewhat. Consider two confocal ellipsoids, i.e., their axis lengths satisfy

$$
a_{1}^{2}-a_{2}^{2}=b_{1}^{2}-b_{2}^{2}=c_{1}^{2}-c_{2}^{2},
$$

where the subscripts 1 and 2 refer to the two ellipsoids. Franx (1988a) showed that two aligned, confocal ellipsoids have the same position angle for all viewing angles. We introduce the triaxiality parameter $T$ by

$$
T=\frac{a^{2}-b^{2}}{a^{2}-c^{2}}=\frac{\epsilon_{2}\left(2-\epsilon_{2}\right)}{\epsilon_{1}\left(2-\epsilon_{1}\right)} .
$$

It follows that all confocal ellipsoids have the same $T$, and all aligned ellipsoids that have the same $T$ can be scaled to be confocal. Thus, equation (2) can be rewritten as

$$
\epsilon=\epsilon\left(\epsilon_{1}, T, \phi, \theta\right), \quad \Gamma_{\text {minor }}=\Gamma_{\text {minor }}(T, \phi, \theta) .
$$

Hence the position angle $\Gamma_{\text {minor }}$ is a function only of the triaxiality parameter $T$ and the viewing angles. This special property has an important consequence with regard to isophotal twists (F88): if the axis ratios of a triaxial galaxy change with distance from the center, then the position angle of the projected galaxy does not necessarily change with radius. If $T$ is constant, the position angle is expected to be constant (if we assume that eq. [5] is valid if the axial ratios change slowly with radius). Thus position-angle twists are caused not by arbitrary changes in the axial ratios, but more specifically, by changes in $T$ with radius.

In Figure 1 we draw the plane of all possible shapes $\left(\epsilon_{1}, \epsilon_{2}\right)$. We show curves of constant $T$. The limiting cases $T=0$ and $T=1$ correspond to oblate and prolate shapes, respectively. It should be noted that the parameter $T$ is quite similar, but not identical, to the triaxiality parameter $Z$, defined by Binney $(1985)$ as $Z=(a-b) /(a-c)$.

In Figure 1 we have indicated two triaxial models, $\mathrm{A}$ and $\mathrm{B}$.

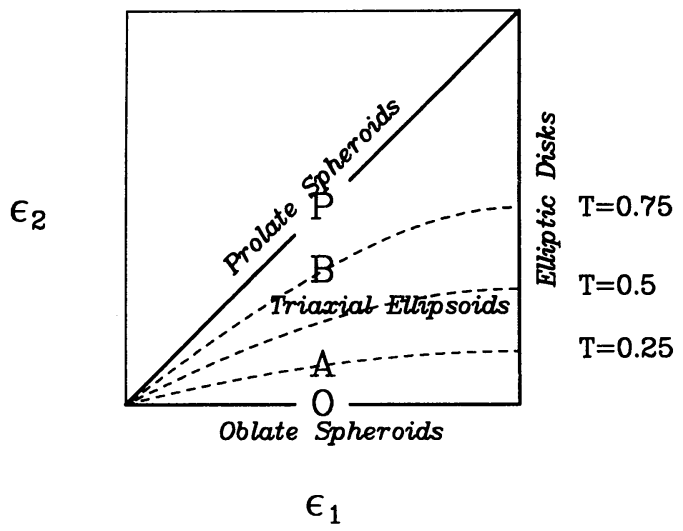

FIG. 1.-Plane of intrinsic shapes $\left(\epsilon_{1}, \epsilon_{2}\right)$. Dashed lines are contours of constant triaxiality $T$. The models $\mathrm{A}, \mathrm{B}, \mathrm{O}$, and $\mathrm{P}$ are indicated (see Table 1 ).
TABLE 1

STANDARD EXAMPLES

\begin{tabular}{ccllll}
\hline \hline Model & $\epsilon_{1}$ & \multicolumn{1}{c}{$\epsilon_{2}$} & \multicolumn{1}{c}{$T$} & $\psi_{\text {int }}$ & \multicolumn{1}{c}{ Shape } \\
\hline A $\ldots \ldots \ldots \ldots \ldots \ldots$ & 0.5 & 0.1 & 0.25 & $17^{\circ}$ & Oblate-triaxial \\
B $\ldots \ldots \ldots \ldots \ldots \ldots$ & 0.5 & 0.34 & 0.75 & $72^{\circ}$ & Prolate-triaxial \\
O $\ldots \ldots \ldots \ldots \ldots \ldots$ & 0.5 & 0 & 0 & $\ldots$ & Oblate \\
P.............. & 0.5 & 0.5 & 1 & $\ldots$ & Prolate \\
\hline
\end{tabular}

The axial ratios are given in Table 1 . The triaxial model $\mathbf{A}$ is near to oblate, and B is near to prolate. In Figures $2 a$ and $2 b$ contours of constant apparent ellipticity are drawn on the unit sphere of projection directions for models A and B. The apparent ellipticity varies between 0 and $\epsilon_{1}$. The minimum occurs along lines of sight in the $(x, z)$-plane, defined by $\tan ^{2} \theta=$ $T /(1-T)$. The maximum apparent ellipticity is reached when looking down the intermediate axis (the $y$-axis). In Figures $2 c$ and $2 d$ we present contours of constant $\Gamma_{\text {minor }}$. The position angle increases slowly from $0^{\circ}$ in the $(x, y)$-plane to $90^{\circ}$ on a section of the $(x, z)$-plane. At two points all contours converge, at the $z$-axis (where the position angle of the projected $z$-axis is degenerate) and at the point where the apparent ellipticity of the galaxy is zero, so that the position angle of the galaxy is degenerate.

\subsection{Projection of Velocity Fields 2.2.1. Apparent Angular Momentum}

Suppose one has measured the complete surface brightness distribution and the complete velocity field, i.e., the apparent radial velocity at all points in the plane of projection. The apparent angular momentum of the projected distribution is defined in the following way: assume a disklike structure with surface density equal to the measured surface density and with velocities perpendicular to the disk equal to the observed radial velocities. The apparent angular momentum is the angular momentum of this structure. Thus, the apparent angular momentum is fully specified by the observed surface brightness distribution and the observed two-dimensional velocity field. Franx (1988b) showed that the apparent angular momentum is parallel to the projection of the intrinsic angular momentum, if figure rotation is absent and if the streaming satisfies the equation of continuity. ${ }^{2}$ The projection of the full three-dimensional velocity field is thus reduced to the projection of the angular momentum vector.

In a general triaxial galaxy, the angular momentum vector is not required to be parallel to the intrinsic short axis, the $z$-axis, but it can lie anywhere in the $(x, z)$-plane (Schwarzschild 1979; Levison \& Richstone 1987; Statler 1987). Define $\psi_{\text {int }}$ as the angle between the $z$-axis and the angular momentum. It is straightforward to calculate the position angle $\Gamma_{\text {kin }}$ of the apparent angular momentum with respect to the projected $z$-axis:

$$
\tan \Gamma_{\mathrm{kin}}=\frac{\sin \phi \sin \psi_{\mathrm{int}}}{-\cos \phi \cos \theta \sin \psi_{\mathrm{int}}+\sin \theta \cos \psi_{\mathrm{int}}} .
$$

In Figures $2 e-2 f$ we show contours of constant $\Gamma_{\text {kin }}$ of models A and B. The measured value of $\Gamma_{\text {kin }}$ is equal to $\psi_{\text {int }}$ when looking down the intermediate axis, and it increases toward the $z$-axis. It reaches $90^{\circ}$ along the thickly drawn contours, and

2 The apparent rotation measured along this axis is not necessarily zero. 


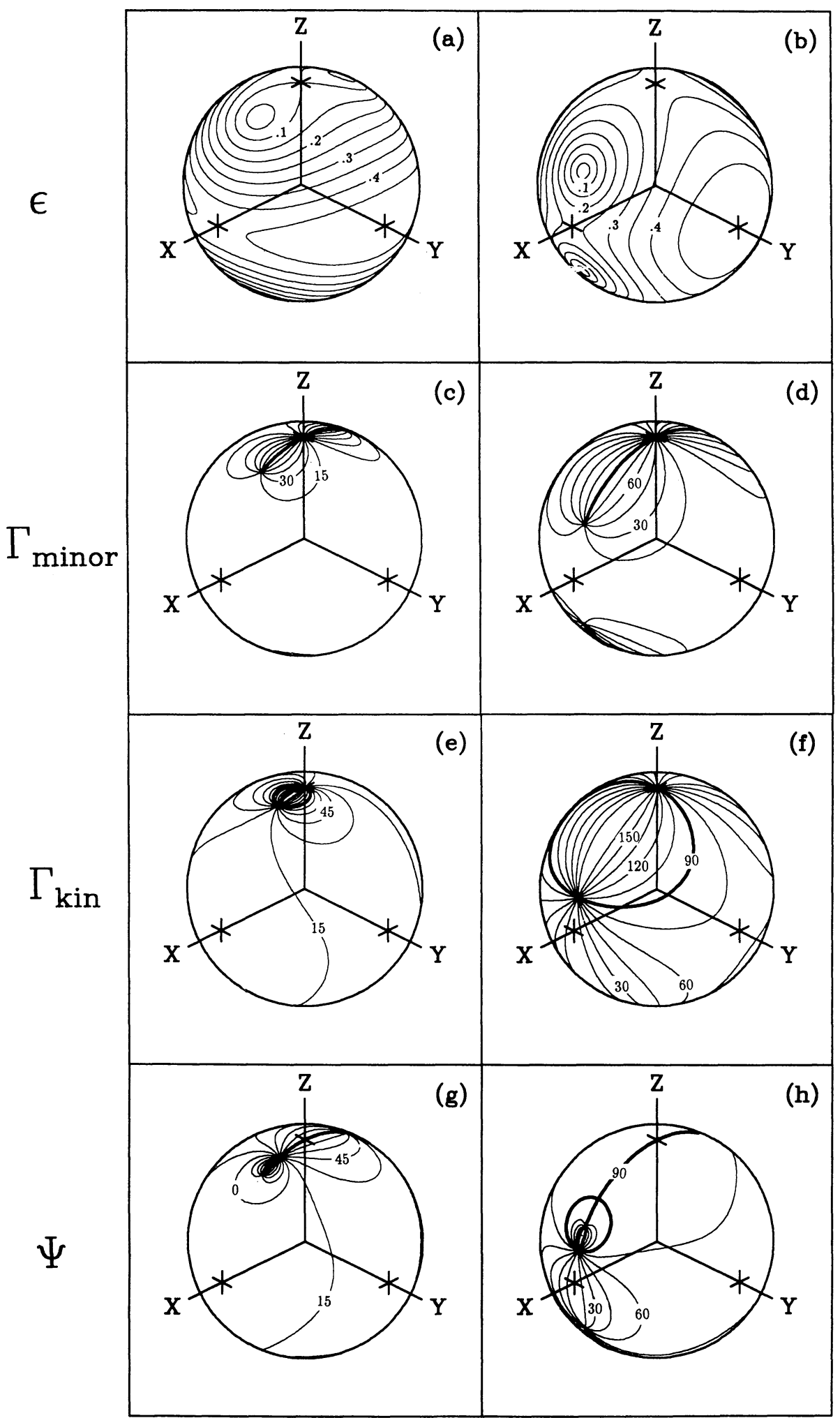

FIG. 2.-Projection of models A and B. Model A (left-hand panel) is oblate-triaxial; model B (right-hand panel) is prolate-triaxial (see Fig. 1 and Table 1). The galaxies are oriented with their longest axis along the $x$-axis and their shortest axis along the $z$-axis. Each viewing angle corresponds to a position on the sphere of projection directions. $(a, b)$ Contours of constant apparent ellipticity $\epsilon$ on the sphere of projection directions. Contours of $\epsilon$ are drawn at intervals of 0.05 . (c, $d$ ) Contours of constant position angle of the minor axis, $\Gamma_{\text {minor }}$, for models $\mathrm{A}$ and $\mathrm{B}$. Contours are drawn at intervals of $10^{\circ}$. The position angle is $0^{\circ}$ in the $(x, y)$-plane. The position angle reaches a maximum of $90^{\circ}$ at a section of the great circle intersecting the $x$-axis and the $z$-axis (thick curves). (e, $f$ ) Contours of constant position angle of the apparent angular momentum, $\Gamma_{k \text { in }}$, for models $A$ and B. Contours are drawn at intervals of $10^{\circ}$. The position angle is $0^{\circ}$ along the $x$-axis, and is equal to $\psi_{\text {int }}$ along the $y$-axis. It increases to $90^{\circ}$ at the thick contours, and reaches $180^{\circ}$ in the section of the great circle intersecting the $x$-axis and the $z$-axis. $(g, h)$ Contours of constant apparent misalignment $\Psi$ for models $\mathrm{A}$ and B. The apparent misalignment follows from subtraction of the position angle of the apparent angular momentum from the position angle of the apparent minor axis (eq. [15]), which are shown in panels $c-f$. Contours are drawn at intervals of $10^{\circ}$. The apparent misalignment is generally $0^{\circ}$ at the $x$-axis, equal to $\psi_{\text {int }}$ along the $y$-axis, and $90^{\circ}$ at the $z$-axis. The apparent misalignment reaches $90^{\circ}$ at thickly drawn curves. 
continues to increase to $180^{\circ}$ at the $(x, z)$-plane. The distribution is not symmetric with respect to the $(x, y)$ - and $(y, z)$ it planes.

,

,

$\Psi$, which is given by

\subsubsection{Apparent Kinematic Misalignment}

An observer cannot determine the angles $\Gamma_{\text {minor }}$ and $\Gamma_{\text {kin }}$ parately, but only their difference, the kinematic misalignment

$$
\sin \Psi=\left|\sin \left(\Gamma_{\text {kin }}-\Gamma_{\text {minor }}\right)\right|, \quad 0^{\circ} \leq \Psi \leq 90^{\circ} .
$$

This angle will depend on the viewing angles, on the shape of the galaxy (through $\Gamma_{\text {minor }}$ ), and on the intrinsic misalignment (through $\Gamma_{\text {kin }}$ ). If $\psi_{\text {int }}=0$, then $\Gamma_{\text {kin }}=0$, and $\Psi=\Gamma_{\text {minor }}$. Hence in this case only the projection of the triaxial shape of the galaxy is relevant. The contours of constant $\Gamma_{\text {minor }}$ in Figures $2 c$ and $2 d$ are also contours of constant $\Psi$ for this special case. If $T=0$, then $\Gamma_{\text {minor }}=0^{\circ}$, and $\Psi=\Gamma_{\text {kin }}$ for $\Gamma_{\text {kin }} \leq 90^{\circ}$ and $\Psi=90^{\circ}-\Gamma_{\text {kin }}>90^{\circ}$. This is the hypothetical case of an oblate galaxy with intrinsic misalignment. Contours of constant $\Gamma_{\text {kin }}$ in Figures $2 e$ and $2 f$ are contours of constant $\Psi$ for such systems. In Figures $2 g$ and $2 h$ we give contours of the kinematic misalignments of models $\mathrm{A}$ and $\mathrm{B}$. The kinematic misalignment is degenerate at six points in the $(x, z)$-plane, two along the direction of the angular momentum of the galaxy and four along the directions from which the galaxy appears round in projection. The kinematic misalignment is not symmetric with respect to the $(x, y)$ - and $(y, z)$-planes.

\section{PROBABILITY DISTRIBUTION OF OBSERVABLES}

For the vast majority of elliptical galaxies we do not know the viewing angles. This implies that it is impossible to apply the results from $\S 2$ directly to derive the intrinsic shape and misalignment of angular momentum for an individual galaxy. Instead, we have to analyze the statistical distribution of the observed parameters for (large) samples of galaxies, assuming that the viewing angles are randomly distributed. In this section we discuss the probability distributions of the relevant observables.

\subsection{Apparent Ellipticity}

Binney and de Vaucouleurs (1981) have derived the probability distribution of $p(\epsilon) d \epsilon$ for the general case of a triaxial ellipsoid. In $\S 1$ of Appendix A we derive equivalent expressions that are more amenable to numerical integration. The moments $\left\langle\epsilon^{n}\right\rangle$ of the apparent ellipticity $\epsilon$ are defined by

$$
\left\langle\epsilon^{n}\right\rangle=\int_{0}^{1} \epsilon^{n} p(\epsilon) d \epsilon .
$$

They can be expressed as combinations of complete elliptic integrals, and can be easily evaluated numerically. Contours of constant $\langle\epsilon\rangle$ in the plane of possible shapes are shown in Figure 3. The first moment $\langle\epsilon\rangle$ is fairly insensitive to $\epsilon_{2}$ for low $\epsilon_{1}$. For small $\epsilon_{1}, \epsilon_{2}$, one obtains (see Fall \& Frenk 1983)

$$
\langle\epsilon\rangle=\frac{2}{3} \epsilon_{1}+O\left(\epsilon_{1}^{2}, \epsilon_{1} \epsilon_{2}, \epsilon_{2}^{2}, \ldots\right) .
$$

Since the mean apparent ellipticity is not strongly dependent on $\epsilon_{2}$, one does not expect strong constraints on the triaxiality of elliptical galaxies from the observed ellipticities.

\subsection{Kinematic Misalignments for Models with Perfect Intrinsic Alignment $\left(\psi_{\text {int }}=0\right)$}

First we consider models which have their intrinsic angular momentum parallel to the intrinsic short axis. For these

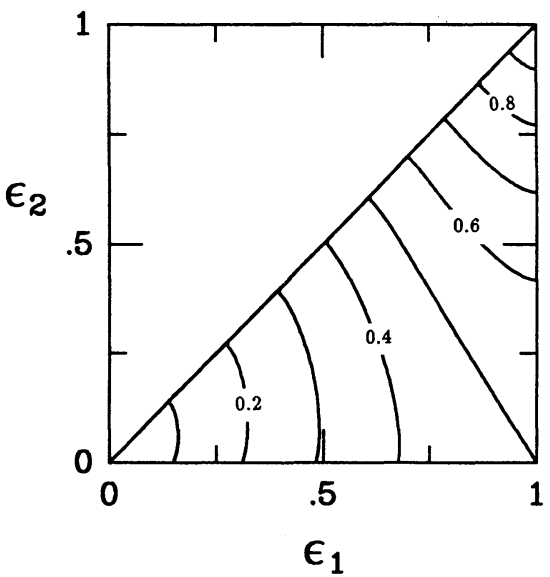

FIG. 3.-Contours of constant mean apparent ellipticity $\langle\epsilon\rangle$ in the plane of all shapes; $\langle\epsilon\rangle$ is the apparent ellipticity averaged over all possible viewing angles. Contours are drawn at intervals of 0.1 . For low $\epsilon_{1},\langle\epsilon\rangle$ is only weakly dependent on $\epsilon_{2}$.

models the kinematic misalignment $\Psi$ is equal to the position angle of the minor axis with respect to the projected $z$-axis, $\Psi=\left|\Gamma_{\text {minor }}\right|$.

We define $p(\Psi, \epsilon)$ as the probability of observing a kinematic misalignment $\Psi$ in the interval $(\Psi, \Psi+d \Psi)$ and an ellipticity $\epsilon$ in the interval $(\epsilon, \epsilon+d \epsilon)$. It can be evaluated by calculation of the corresponding area on the unit sphere of projection directions. This calculation is straightforward to do numerically. In $\S 2$ of Appendix A we derive an analytic expression for $p(\epsilon, \Psi)$, which is useful for verifying the numerical results. The resulting expression is complex, and is not explicit in terms of $\epsilon, \Psi$. In Figure 4 we show the probability distributions for two models with the shapes of models A and B, but no intrinsic misalign-

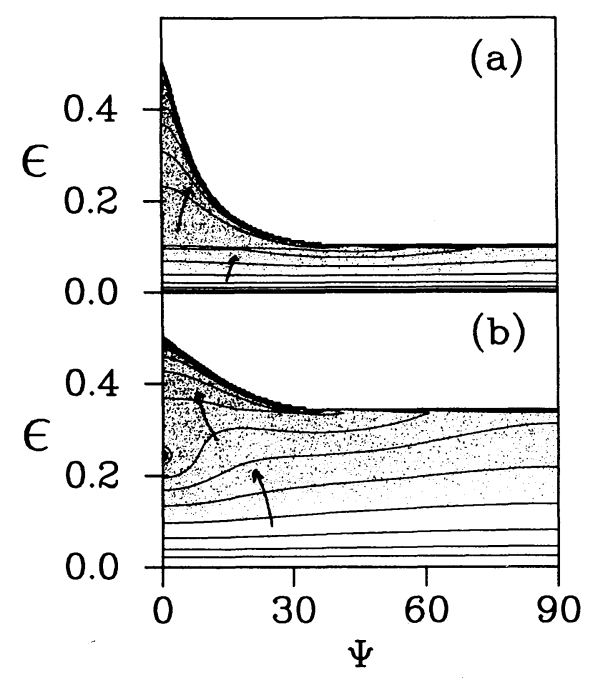

FIG. 4.-Probability distributions $p(\epsilon, \Psi)$ of apparent misalignment $\Psi$ and ellipticity $\epsilon$ for triaxial models with perfectly aligned angular momenta. The shapes of the models in $(a)$ and $(b)$ are those of models A (oblate-triaxial) and B (prolate-triaxial), respectively, but the intrinsic misalignment $\psi_{\mathrm{int}}$ is taken to be zero. Contours are drawn at logarithmic intervals of $\Delta{ }^{10} \log p=\frac{1}{4}$. The probability distribution increases along the arrows. The maximum apparent ellipticity at $\Psi=90^{\circ}$ is $\epsilon_{2}$. The corresponding viewing angle is along the $z$-axis. The main difference between the oblate-triaxial model and the prolate-triaxial model is the absence of flat galaxies with large misalignments in the former case. 


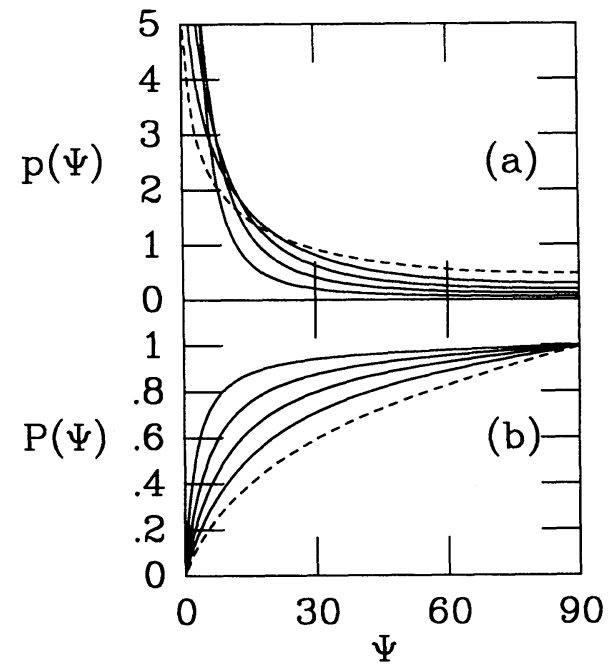

Fig. 5.- (a) Probability distribution $p(\Psi)$ for galaxies with triaxialities $T=0.2,0.4, \ldots, 1$. The dashed curve is for $T=1$. The higher the value of $T$, the higher the probability at $\Psi=90^{\circ}$. The probability distributions have been normalized to give a mean of 1 over the relevant interval. (b) Corresponding cumulative probability distribution $P(\Psi)$. The lowest curve has the highest $T$.

ment. The probability distribution does not depend strongly on $\Psi$ for $\epsilon<\epsilon_{2}$, but is strongly dependent on $\Psi$ for $\epsilon>\epsilon_{2}$. The largest misalignments are always found at small ellipticities. The most important difference between the distributions in Figures $4 a$ and $4 b$ is at intermediate apparent ellipticity and high misalignment. The first distribution is zero in this region, whereas the second distribution (which applies to the galaxy which is more prolate) is positive.

The probability distribution $p(\Psi) d \Psi$ is derived in $\S 2$ of Appendix A. Since the kinematic misalignment $\psi$ depends only on $T$ and the viewing angles, it follows that $p(\Psi) d \Psi$ depends only on $T$.

In Figure 5 we have plotted curves of $p(\Psi)$ and the cumulative probability $P(\Psi)$ of finding $\Psi$ between zero and a specified value,

$$
P(\Psi)=\int_{0}^{\Psi} p\left(\Psi^{\prime}\right) d \Psi^{\prime} .
$$

The extreme case $T=0$ corresponds to oblate models, and has $p(\Psi)=\delta(\Psi)$. The extreme $T=1$ corresponds to prolate models. Note that all the distributions $p(\Psi)$ have a singularity at $\Psi=0$. The value of $p(\Psi)$ at $\Psi=90^{\circ}$ increases with increasing $T$.

We define the moments $\left\langle\Psi^{i}\right\rangle$ of the observed misalignment. These are equal to the value of $\Psi^{i}$ averaged over all viewing angles. In Figure $6 a$ we show the mean observed misalignment $\langle\Psi\rangle$ and the standard deviation $\sigma(\Psi)=\left(\left\langle\Psi^{2}\right\rangle-\langle\Psi\rangle^{2}\right)^{1 / 2}$ as a function of $T$. Surprisingly, the mean observed misalignment is almost a linear function of the triaxiality parameter $T$. This has an important implication: it shows that the measurement of the mean misalignment of a sample of galaxies is relatively simply related to the mean triaxiality $\langle T\rangle$ of that sample, if this class of models is applicable. The standard deviation in the distribution of observed misalignments is high, higher than the mean observed misalignment for almost all $T$.

As we have seen in Figure 4, the probability distribution of $\Psi$ depends strongly on the measured apparent ellipticity. We have illustrated this effect in Figure $6 b$, where we plot $\langle\epsilon \Psi\rangle /(\langle\epsilon\rangle\langle\Psi\rangle)-1$ against $T$. This quantity is a measure of the correlation of the apparent ellipticity with observed kinematic misalignment. It is negative, because the ellipticity and kinematic misalignment are anticorrelated (at low ellipticities we measure the highest misalignments). The effect is the strongest at low $T$. This shows that if we want to use the moment analysis to derive the mean triaxiality from the mean kinematic misalignment, we have to make sure that the sample is unbiased in ellipticity. A bias in ellipticity in the data sample can produce a bias in the mean misalignment, given the correlation between the two.

\subsubsection{Observed Kinematic Misalignment for Galaxies with Aligned Angular Momenta}

For many galaxies, only the radial velocities along the major and minor axes have been measured. For such systems, the misalignment is usually determined by

$$
\tan \Psi_{\text {obs }}=\frac{v_{\text {minor }}}{v_{\text {major }}},
$$

where $v_{\text {minor }}$ and $v_{\text {major }}$ are the velocity amplitudes along the minor and major axes, respectively. The angle $\Psi_{\text {obs }}$ can deviate from the "real" misalignment angle, $\Psi$, between the apparent angular momentum and the apparent short axis. Here we will investigate what errors can be introduced by this approximation.

This type of analysis has been done before by Binney (1985), who assumed that the streaming was along contours of constant density. There is no particular reason that this should be the case for ellipticals. Hence the results will be modeldependent, i.e., they will depend on the details of the streaming motions within ellipticals. We will use the more general models from Franx (1988b), which have streaming along similar ellipses in planes perpendicular to the short axis. The ellipses can have arbitrary shapes and orientations with respect to the density distribution. The streaming satisfies the equation of continuity. We constructed models with a luminosity density

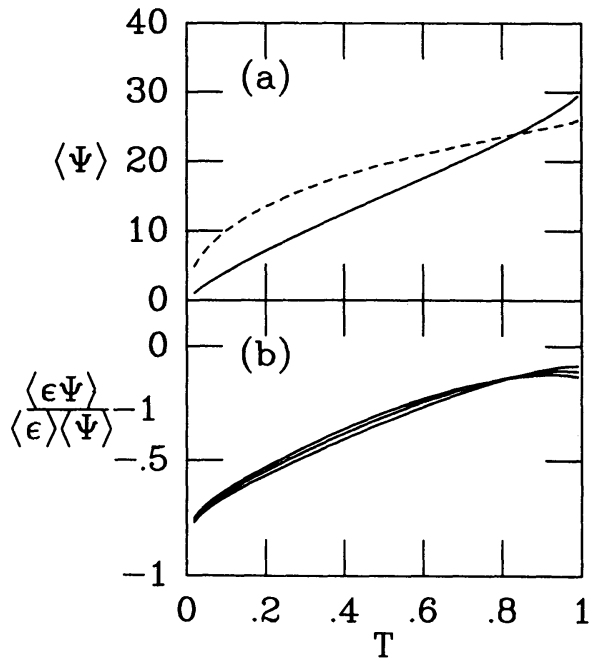

Fig. 6.-(a) Mean apparent misalignment $\langle\Psi\rangle$ as a function of $T$ for models with perfect intrinsic misalignment. $\langle\Psi\rangle$ is the misalignment averaged over all viewing angles. Surprisingly, $\langle\Psi\rangle$ is an almost linear function of $T$. The dashed curve is the dispersion in apparent misalignment $\sigma(\Psi)$. It is comparable to $\langle\Psi\rangle$ over the whole interval of $T$. These functions are completely independent of the value of $\epsilon_{1}$. (b) Correlation between the apparent ellipticity $\epsilon$ and the apparent misalignment $\Psi$ as a function of $T$. Curves are drawn for $\epsilon_{1}=0.1,0.3$, and 0.5 . 
decreasing as $r^{-3}$ and streaming velocities independent of radius. We considered three special cases of the streaming models: First, we analyzed Binney's streaming models, in which the streaming is along contours of constant density. It is straightforward to derive for this case

$$
\frac{v_{\text {minor }}}{v_{\text {major }}}=\frac{\tan \Gamma_{\text {minor }}}{1-\epsilon},
$$

where $\Gamma_{\text {minor }}$ is the position angle of the apparent short axis with respect to the projected $z$-axis. Since the angular momentum points along the intrinsic short axis, the observed kinematic misalignment $\Psi_{\text {obs }}$ is simply related to the misalignment $\Psi$ of the apparent angular momentum.

Second, we constructed models with circular streamlines. We did not find simple relations for the observed kinematic misalignment. As a third kinematic model, we considered the case in which the streaming is along ellipses with the same axial ratio as the isodensity contours in the $(x, y)$-plane, but with an elongation perpendicular to the elongation of the isodensity contours. Such an orientation is typical of streaming in nonrotating triaxial potentials, and may therefore be more appropriate for triaxial models without figure rotation than the previous streaming models.

The probability distributions of $p\left(\Psi_{\mathrm{obs}}, \epsilon\right)$ do not differ greatly from the distribution $p(\Psi, \epsilon)$, which applies if the full velocity field is used. We illustrate the differences in Figure 7. In Figure $7 a$ the integrated probability distribution $p\left(\Psi_{\text {obs }}\right)$ is shown for the three streaming models with $\epsilon_{1}=0.4$ and $T=0.56$. Figure $7 b$ shows the mean apparent misalignment $\langle\Psi\rangle$ as a function of $T$. There are differences between the models, but the effects are not overwhelming. Thus the observed misalignment $\Psi_{\text {obs }}$ can be used as the real misalignment angle $\Psi$.
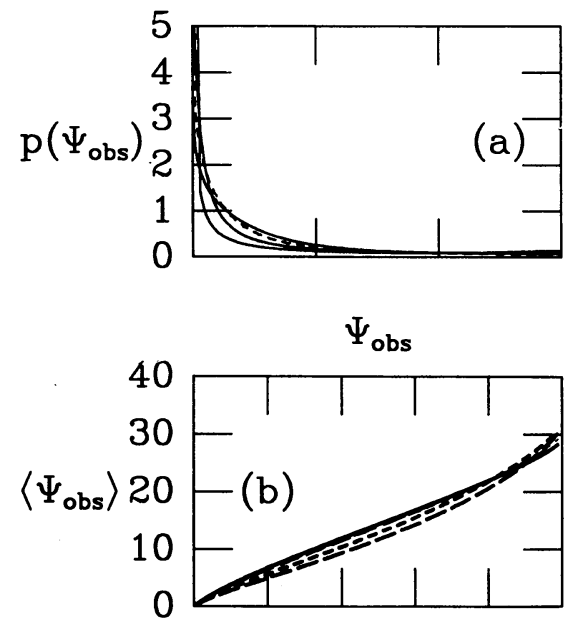

$\mathrm{T}$

FIG. 7.- (a) Distribution $p\left(\Psi_{\mathrm{obs}}\right)$ for the models with streaming parallel, circular, and perpendicular to the shapes of the galaxy. The dashed curve is the distribution $p(\Psi)$ for a model with the same shape. (b) Mean observed misalignment $\left\langle\Psi_{\text {obs }}\right\rangle$ as a function of triaxiality. The continuous curve is for models with parallel streaming, the short-dashed curve for circular streaming, and the long-dashed curve for perpendicular streaming. These curves apply to galaxies with $\epsilon_{1}=0.5$. The equivalent curves for galaxies with $\epsilon_{1}=0.1$ coincide with the thick continuous curve. Note that the differences are not large in both cases, and thus $\left\langle\Psi_{\text {obs }}\right\rangle \approx\langle\Psi\rangle$.

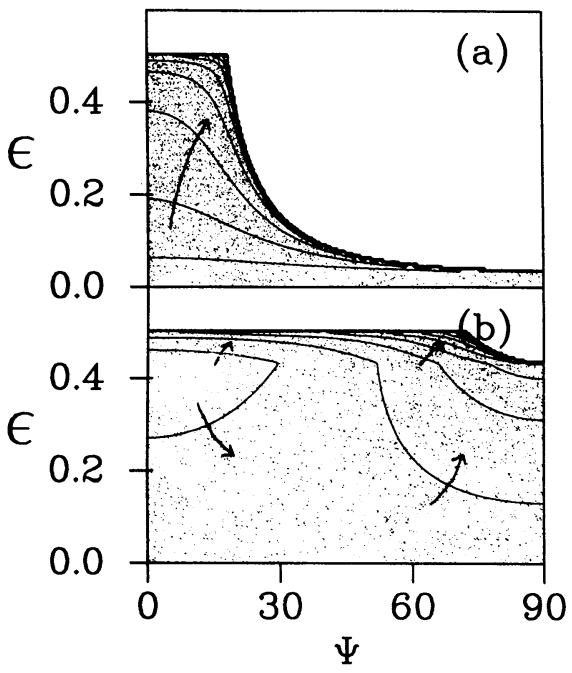

Fig. 8.-Probability distributions $p(\epsilon, \Psi)$ of apparent misalignments and ellipticities for oblate models with misaligned angular momenta. The intrinsic misalignments in $(a)$ and $(b)$ are those of models A and B $\left(\psi_{\mathrm{int}}=18^{\circ}, 72^{\circ}\right.$, respectively), but the triaxiality $T$ is equal to zero. Contours are drawn at logarithmic intervals of $\Delta^{10} \log p=\frac{1}{4}$. The probability distribution increases along the arrows. The probability distribution is singular along the curve of maximum $\epsilon$ for fixed $\Psi$. The maximum apparent misalignment at $\epsilon=\epsilon_{1}$ equals $\psi_{\text {int }}$, the intrinsic misalignment. Many of the viewing angles project near to this point. The maximum apparent ellipticity equals $1-\left[\sin ^{2} \psi_{\text {int }}\left(1-\epsilon_{1}\right)^{2}+\right.$ $\left.\cos ^{2} \psi_{\text {int }}\right]^{1 / 2}$ at $\Psi=90^{\circ}$.

\subsection{Kinematic Misalignments for Models with Intrinsic Misalignment}

Next we present the probability distributions of $(\epsilon, \Psi)$ for models with intrinsic misalignments of the angular momentum and the short axis. The most extreme of such models are triaxial, near-oblate, with large intrinsic misalignments. For such models the apparent misalignments are mainly caused by the intrinsic misalignment and not by the projection of the triaxial shape. In the following subsection, we consider oblate models with intrinsic misalignments. Such models are not realistic, since exactly oblate models will not have intrinsic misalignments. They are useful, however, because they can be contrasted with the triaxial models discussed above which had no intrinsic misalignments and for which all apparent misalignments were due to projection effects. Furthermore, the resulting probability distributions should be similar to those of nearly oblate triaxial galaxies, which can have intrinsic misalignments (e.g., Statler 1987).

\subsubsection{Oblate Models with Intrinsic Misalignments}

For oblate models the apparent short axis coincides with the projection of the intrinsic short axis, and hence $\Gamma_{\text {minor }}=0$. We assume that the full velocity field has been mapped, and the apparent misalignment been determined. The probability distribution $p(\epsilon, \Psi)$ is easily evaluated numerically. An analytic result is given in $\S 3$ of Appendix $A$.

Figure 8 shows the probability distribution for two values of $\psi_{\text {int }}$. The distribution is singular along the curve of maximum $\epsilon$ for a given $\Psi$. There are two special points: first, the point of maximum misalignment at $\epsilon=\epsilon_{1}$. The misalignment equals the intrinsic misalignment at this point. The corresponding viewing angle is along the $y$-axis. The second point of maximum ellipticity at $90^{\circ}$ misalignment coincides with a viewing angle along the intrinsic angular momentum.

The probability distribution $p(\Psi) d \Psi$ is derived in $\S 3$ of 


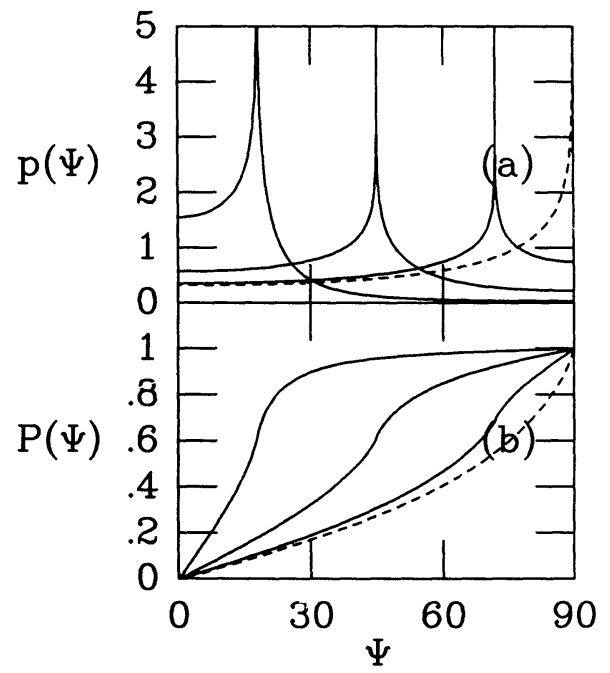

FIG. 9.-(a) Probability distribution $p(\Psi)$ for oblate galaxies with intrinsic misalignments $\psi_{\text {int }}=18^{\circ}, 45^{\circ}, 72^{\circ}, 90^{\circ}$. The distributions show a singularity at $\Psi=\psi_{\text {int }}$. The dashed line corresponds to $\psi_{\text {int }}=90^{\circ}$. The probability distributions have been normalized to give a mean of 1 over the relevant interval. (b) Corresponding cumulative probability distribution $P(\Psi)$. The lowest curve has the highest $\psi_{\text {int }}$.

Appendix A. We show examples of the resulting distribution in Figure 9. All the distributions $p(\Psi)$ show a singularity at $\Psi=$ $\psi_{\text {int }}$. In that aspect the distributions are distinctly different from the distributions for the misalignments of triaxial systems without intrinsic misalignment, which showed a singularity at $\Psi=0$ for all triaxialities (see $\S 3.2$ ). The probability distribution $p(\Psi)$ is larger than zero for all $\Psi$ for all $\psi_{\text {int }}>0$.

We have calculated the mean apparent misalignment $\langle\Psi\rangle$ and the standard deviation $\sigma(\Psi)$ numerically for these distributions, and we show the results in Figure 10a. The mean observed misalignment increases almost linearly with $\psi_{\text {int }}$ for small $\psi_{\text {int }}$, and flattens out at large $\psi_{\text {int }}$. Note that the mean

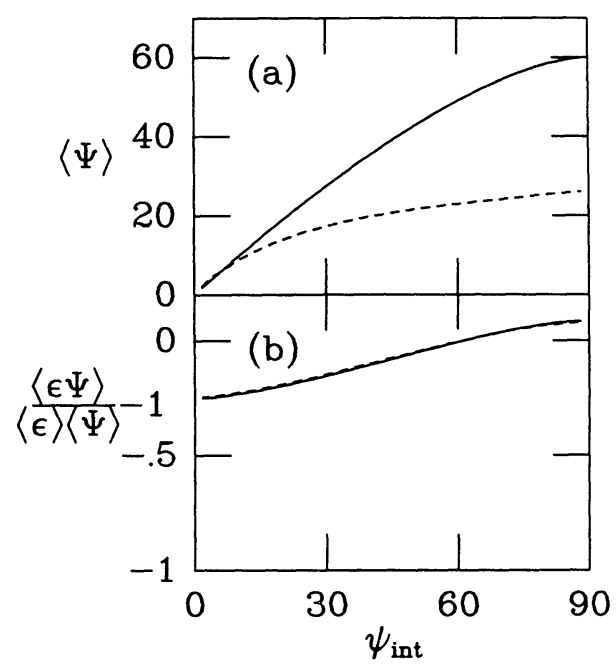

Fig. 10.- (a) Mean apparent misalignment $\langle\Psi\rangle$ as a function of $\psi_{\text {int }}$ for oblate models with intrinsic misalignment. The mean apparent misalignment reaches as high as $60^{\circ}$. The dashed curve is the dispersion in apparent misalignment, $\sigma(\Psi)$. These functions are completely independent of the value of $\epsilon_{1} .(b)$ Correlation between apparent ellipticity $\epsilon$ and apparent misalignment $\Psi$ as a function of $\psi_{\text {int }}$ for $\epsilon=0.4$. The dashed curve for $\epsilon=0.2$ almost coincides with the curve for $\epsilon=0.4$ observed misalignments can become significantly larger than for the previous case of triaxial aligned models. There is again a significant dispersion $\sigma(\Psi)$ compared with the mean $\Psi$, but the ratio $\sigma(\Psi) /\langle\Psi\rangle$ is about half that for the triaxial aligned models.

In Figure $10 b$ the correlation between $\Psi$ and $\epsilon$ is shown. The correlation is much smaller than for the case of triaxial aligned models. Hence a bias in apparent ellipticity in the data sample is less important if this type of model is used.

\subsubsection{Kinematic Misalignments for Triaxial Models with Misaligned Angular Momenta}

The most general type of model is a triaxial galaxy with intrinsic misalignment. The angular momentum is required to lie in the $(x, z)$-plane, and the angle between the $z$-axis and the angular momentum is $\psi_{\text {int }}$. In Figures $2 g$ and $2 h$ we show the apparent misalignment as a function of the viewing angles. The calculation of the probability distribution $p(\epsilon, \Psi)$ is too laborious analytically; hence we have calculated the probability distribution numerically. Results for models A and B are shown in Figure 11. There are at least three pairs of special viewing angles on the sphere of projection directions: one pair along the direction of the angular momentum and two pairs where the galaxy appears round. The misalignment is not defined at those points.

We have integrated $p(\epsilon, \Psi)$ to obtain the probability distribution $p(\Psi) d \Psi$. The distribution $p(\Psi)$ depends only on the triaxiality parameter $T$ and the intrinsic misalignment $\psi_{\text {int }}$, and is independent of the intrinsic ellipticity $\epsilon_{1}$. Figure 12 shows the resulting distributions for the two models of Figure 11. The probability distribution has a singularity at $\Psi=\psi_{\text {int }}$, and is nonzero for all $\Psi$, if $0^{\circ}<\psi_{\text {int }}<90^{\circ}$. We have also shown the probability distribution for three galaxies with $\psi_{\text {int }}=45^{\circ}$ and very different $T$. It is remarkable-and unfortunate - that the probability distributions are virtually identical. It is straightforward to prove that

$$
p\left(\Psi \mid T, \psi_{\text {int }}\right)=p\left(90^{\circ}-\Psi \mid 1-T, 90^{\circ}-\psi_{\text {int }}\right) .
$$

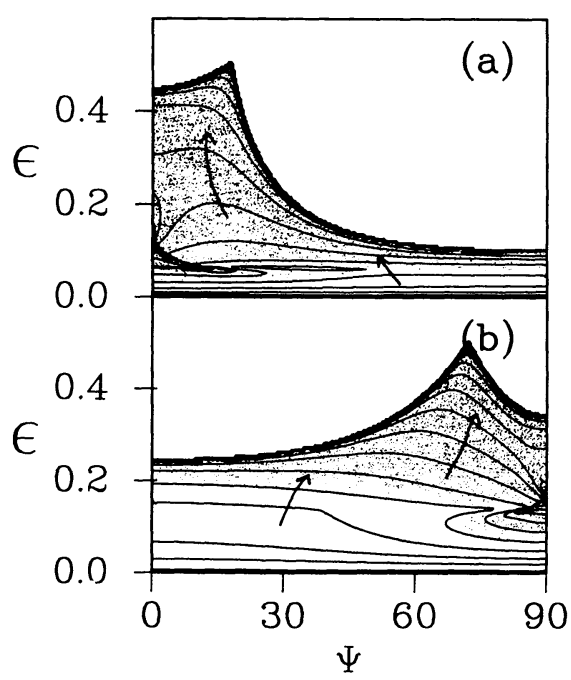

Fig. 11.-Probability distributions $p(\epsilon, \Psi)$ of apparent misalignments and ellipticities for models $\mathrm{A}$ and $\mathrm{B}$, which are triaxial models with misaligned angular momenta (see Fig. 2 and Table 1). These are the most general models to be considered. Contours are drawn at logarithmic intervals of $\Delta^{10} \log p=$ $\frac{1}{4}$. The probability distribution increases along the arrows. (a) Model A, oblatetriaxial with small intrinsic misalignment. (b) Model B, prolate-triaxial with large intrinsic misalignment. 


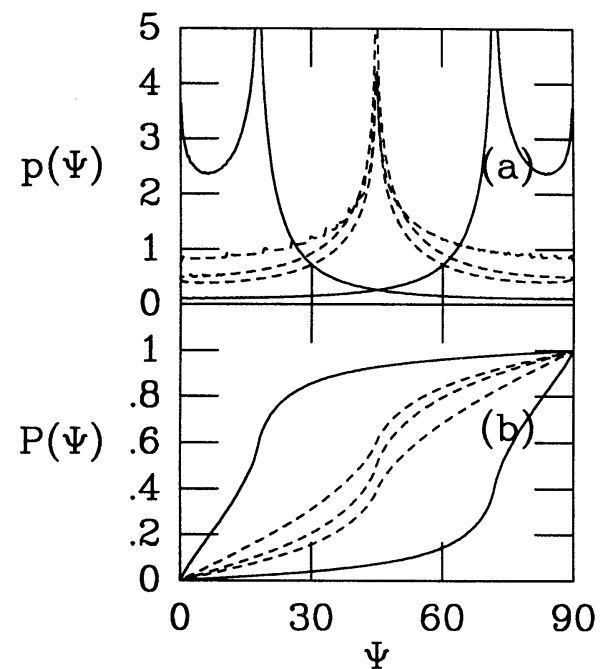

Fig. 12.-Probability distribution $p(\Psi)$ for triaxial models with intrinsic misalignments. The continuous curves apply to models $A$ and $B$. The probability distributions are singular at $\Psi=\psi_{\mathrm{int}}$. The dashed curves are for special models with misalignments $\psi_{\text {int }}=45^{\circ}$ and triaxiality $T=0,0.5,1$. The probability distribution is almost independent of $T$ for this special value of $\psi_{\mathrm{int}}$. (b) Cumulative probability distribution $P(\Psi)$ for the models shown in $(a)$.

An obvious example is $T=0$ and $\psi_{\text {int }}=0$, which is an oblate model with the angular momentum along the intrinsic short axis and which has zero apparent misalignment. By equation (13), the complementary model is a prolate model with the angular momentum along the intrinsic long axis, which has $90^{\circ}$ misalignment for all viewing angles.

Figure $13 a$ shows the mean observed misalignment angle $\langle\Psi\rangle$ as a function of $T$ and $\psi_{\text {int }}$. Equation (13) implies

$$
\langle\Psi\rangle\left(T, \psi_{\text {int }}\right)=90^{\circ}-\langle\Psi\rangle\left(1-T, 90^{\circ}-\psi_{\text {int }}\right) .
$$

This produces the symmetry in the contours. Figure $13 a$ shows clearly that the mean observed misalignment increases with increasing $T$ and increasing $\Psi$ for all values of $T$ and $\Psi$. This has the important consequence that we can find upper limits on the intrinsic mean triaxiality by using models with zero intrinsic misalignment. Second, the mean observed misalign- ment depends only weakly on the triaxiality parameter for intermediate misalignments $\left(20^{\circ}<\psi_{\text {int }}<70^{\circ}\right)$. Only for $\psi_{\text {int }} \sim$ $0^{\circ}$ or $\psi_{\text {int }} \sim 90^{\circ}$ is the mean apparent misalignment strongly dependent on $T$.

Figure $13 b$ shows the correlation coefficient $c$ of the apparent misalignment with the apparent ellipticity, $c=$ $(\langle\Psi \epsilon\rangle-\langle\Psi\rangle\langle\epsilon\rangle) /[\sigma(\Psi) \sigma(\epsilon)]$, for $\epsilon_{1}=0.4$. The correlation coefficient is strongly negative at low $\psi_{\text {int }}$ and $T$, and is high at high $\psi_{\text {int }}$ and $T$. This shows again that a sample biased in ellipticity can produce a biased distribution of apparent misalignments.

\section{DATA COMPILATION}

There are two basic observational constraints on the models: (1) the distribution of observed ellipticities and (2) the distribution of observed ellipticities and misalignments. These two distributions have to be considered separately because there is not a complete sample of galaxies with kinematic misalignments. As we shall see later, the current sample is strongly biased toward round galaxies.

\subsection{Distribution of Apparent Ellipticities}

Contradictory results on the frequency distribution of apparent ellipticities have been reported, as can be seen, for example, in the review by Schechter (1987). A reevaluation of the frequency distribution is justified by the extensive data on intensity, ellipticity, and position-angle profiles that have recently been derived from analyses of high signal-to-noise CCD images (Djorgovski 1985; Jedrzejewski 1987; Bender \& Möllenhof 1987; Bender, Döbereiner, \& Möllenhof 1988; Franx, Illingworth, \& Heckman 1989a; Peletier et al. 1990). The $R$-band profiles given by these authors were converted to the standard Johnson $R$ band. The ellipticity for each galaxy was then determined at the isophotal radius of $\mu_{R}=20.5 \mathrm{mag}$ $\operatorname{arcsec}^{-2}$, where the ellipticities are generally least influenced by seeing and noise. To reduce the noise, the listed ellipticities for each galaxy were averaged within a radial interval, which spanned a range in surface brightness of $1 \mathrm{mag} \operatorname{arcsec}^{-2}$. Special caution is needed to avoid systematic effects at small ellipticities, where noise tends to systematically increase the
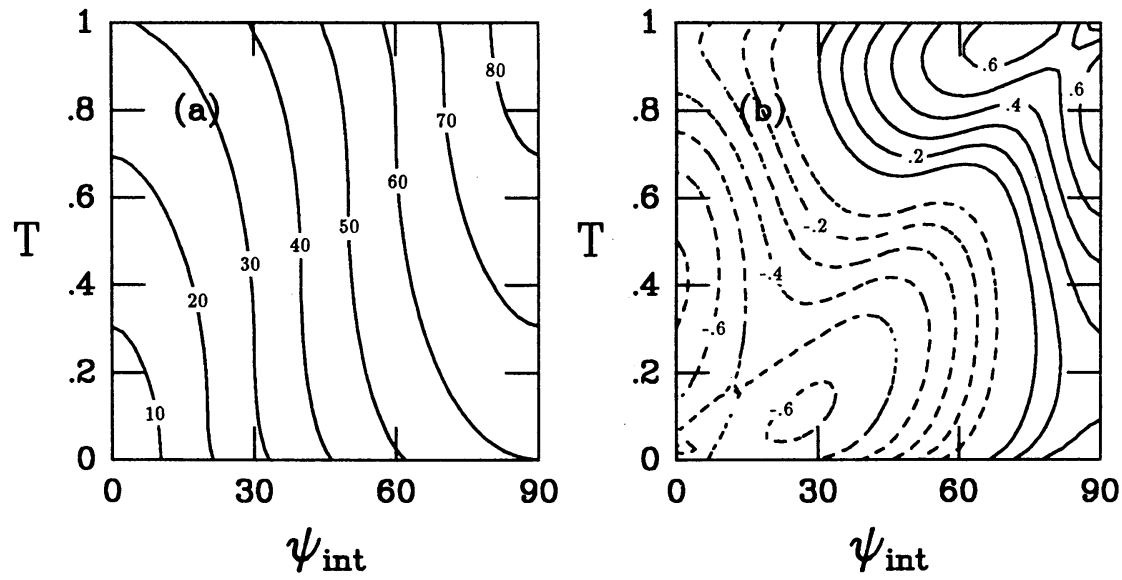

FIG. 13. $-(a)$ Mean apparent misalignment $\langle\Psi\rangle$ as a function of $\psi_{\text {int }}$ and $T$. This is the apparent misalignment averaged over all viewing angles. Contours are

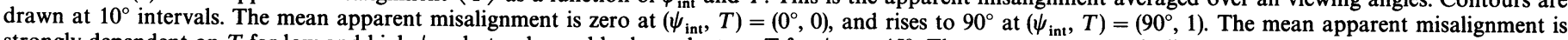

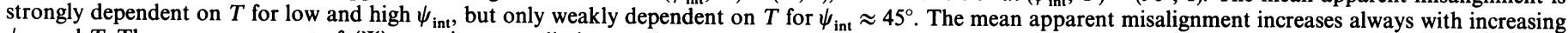

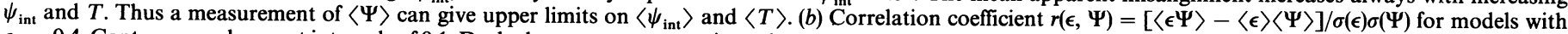
$\epsilon_{1}=0.4$. Contours are drawn at intervals of 0.1 . Dashed contours are negative values. 


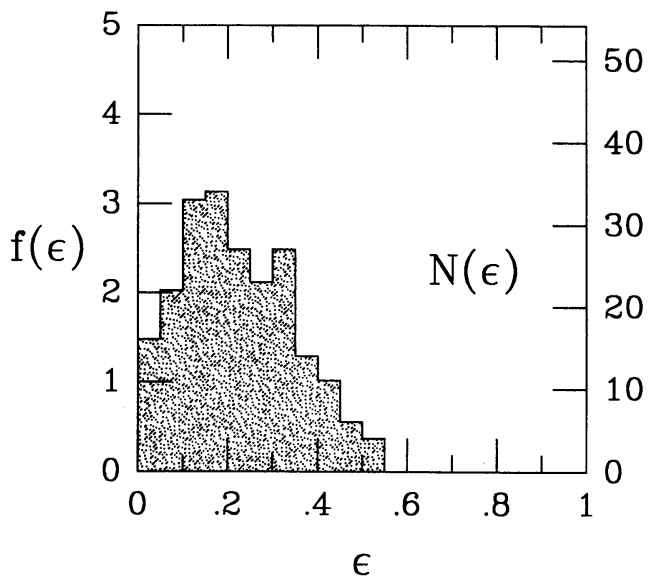

FIG. 14.-Histogram of apparent ellipticities for the CCD sample. The ellipticity is taken at an isophotal radius $\mu_{R}=20.5 \mathrm{mag} \mathrm{arcsec}-2$. There is a relative paucity of nearly round galaxies.

ellipticity. Therefore, we averaged the vectors

$$
\boldsymbol{x}_{i}=\epsilon_{i}\left(\begin{array}{c}
\sin 2 \Gamma_{i} \\
\cos 2 \Gamma_{i}
\end{array}\right),
$$

where $\Gamma$ is the position angle, and $\Gamma_{i}$ and $\epsilon_{i}$ are the individual measurements. The mean ellipticity is defined as the length of the averaged vectors by

$$
\langle\epsilon\rangle=\frac{1}{N} \sqrt{\left(\sum \epsilon_{i} \sin 2 \Gamma_{i}\right)^{2}+\left(\sum \epsilon_{i} \cos 2 \Gamma_{i}\right)^{2}} .
$$

We show the frequency distribution of the apparent ellipticities in Figure 14 and Table 2. The distribution has a peak at $\epsilon \approx 0.2$. The total number of galaxies in the sample is 217 . The mean ellipticity is $\langle\epsilon\rangle=0.22$, and the standard deviation is $\sigma(\epsilon)=0.12$. The frequency distribution is significantly different from that shown in Figure 15a, which is based on the RC2 ellipticities (Binney \& de Vaucouleurs 1981; de Vaucouleurs, de Vaucouleurs, \& Corwin 1976, hereafter RC2), but is similar to those presented by Fasano \& Vio (1991) and Porter, Schneider, \& Hoessel (1991). We tested whether the difference between the current data and the RC2 sample could be due to our particular sample or to differences in definition. One clue was that the frequency distributions for galaxies common to both samples were also inconsistent. The difference persisted if we used ellipticities determined at $\mu_{R}=23.5 \mathrm{mag} \operatorname{arcsec}^{-2}$ (which corresponded to the fiducial radius of $\mu_{B}=25.0 \mathrm{mag}$ $\operatorname{arcsec}^{-2}$ for the RC2 data). Hence the difference was not due to a systematic difference in the radius at which the ellipticity was determined. We show a scatter diagram of the ellipticities from the CCD data compared with the RC2 ellipticities in Figure $15 b$. The RC2 ellipticities have large systematic errors and are strongly biased toward low values for nearly round galaxies. In retrospect, this should not have come as a surprise, since the RC2 ellipticities are derived from a variety of sources and are mostly based on eye estimates.

A third independent source of data was the work of Faber et al. (1989, hereafter 7SAM), who measured ellipticities for a complete sample of ellipticals. This sample is better defined than our CCD sample, but unfortunately the ellipticities are still eye measures. We compare the CCD data and the 7SAM
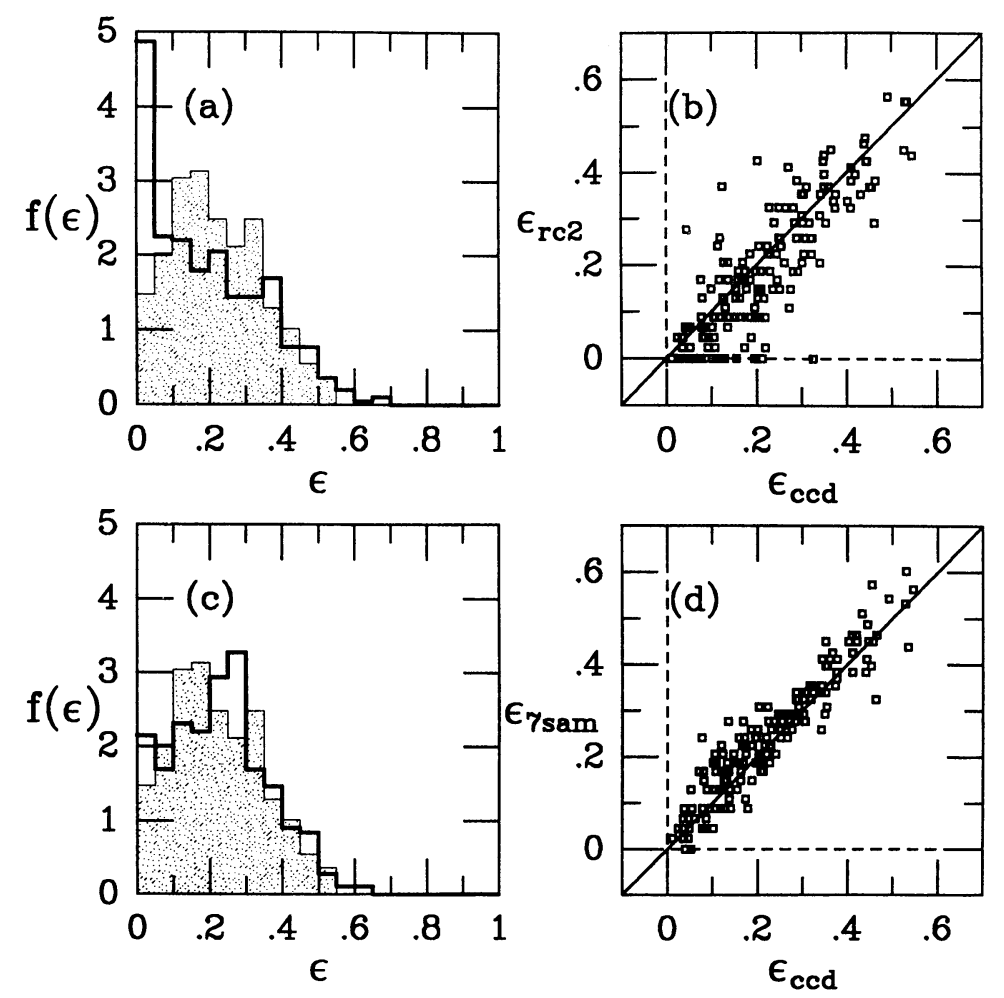

FIG. 15.- (a) Comparison of the histogram of apparent ellipticities from the RC2 (thick line) and our CCD sample (hatched area). Note the abundance of round galaxies in the RC2 data, which is due to systematic measurement errors in the RC2 data. (b) Comparison of ellipticities from the RC2 and CCD sample for all galaxies in common. Many galaxies with nonzero ellipticity have zero ellipticity in the RC2. (c) Comparison of the ellipticities from 7SAM (thick line) and the CCD sample (hatched area). (d) Ellipticities from the 7SAM and the CCD sample for the galaxies in common. The comparison is good, apart from a small systematic effect at $\epsilon_{7 \mathrm{sAM}} \approx 0.2$. 
TABLE 2

Histogram OF OBSERVEd Ellipticities

\begin{tabular}{|c|c|c|c|c|}
\hline \multirow[b]{2}{*}{$\epsilon$} & \multicolumn{2}{|c|}{ CCD SAMPLE } & \multicolumn{2}{|c|}{ 7SAM SAMPLE } \\
\hline & $N$ & $f(\epsilon)$ & $N$ & $f(\epsilon)$ \\
\hline $0.00-0.05 \ldots \ldots$ & 16 & 1.48 & 38 & 2.15 \\
\hline $0.05-0.10 \ldots$ & 22 & 2.03 & 30 & 1.70 \\
\hline $0.10-0.15 \ldots$ & 33 & 3.04 & 41 & 2.32 \\
\hline $0.15-0.20 \ldots \ldots \ldots \ldots \ldots \ldots \ldots$ & 34 & 3.13 & 39 & 2.20 \\
\hline $0.20-0.25 \ldots \ldots \ldots \ldots \ldots \ldots$ & 27 & 2.49 & 52 & 2.94 \\
\hline $0.25-0.30 \ldots \ldots \ldots \ldots \ldots \ldots \ldots$ & 23 & 2.12 & 58 & 3.28 \\
\hline $0.30-0.35 \ldots \ldots \ldots \ldots \ldots \ldots \ldots$ & 27 & 2.49 & 30 & 1.70 \\
\hline $0.35-0.40 \ldots \ldots \ldots \ldots \ldots \ldots \ldots$ & 14 & 1.29 & 26 & 1.47 \\
\hline $0.40-0.45 \ldots \ldots \ldots \ldots \ldots \ldots \ldots$ & 11 & 1.01 & 16 & 0.90 \\
\hline $0.45-0.50 \ldots \ldots \ldots \ldots \ldots \ldots \ldots$ & 6 & 0.55 & 15 & 0.85 \\
\hline $0.50-0.55 \ldots \ldots \ldots \ldots \ldots \ldots \ldots$ & 4 & 0.37 & 5 & 0.28 \\
\hline $0.55-0.60 \ldots \ldots \ldots \ldots \ldots \ldots \ldots$ & 0 & 0.00 & 2 & 0.11 \\
\hline $0.60-0.65 \ldots \ldots \ldots \ldots \ldots \ldots \ldots$ & 0 & 0.00 & 2 & 0.11 \\
\hline
\end{tabular}

data in Figure $15 c$; the histograms are rather similar, and there is no significant difference according to the KolmogorovSmirnov test. The 7SAM data are slightly biased, however, as is shown in Figure 15d. This is probably an artifact of the method that 7SAM used to measure the ellipticities. The effect is not dramatic, and hence these data constitute a good comparison sample.

\subsection{Dependence of Apparent Ellipticity on Other Observables}

Does the distribution of apparent ellipticity depend on other observables? This question has been addressed before by several authors, with mostly negative or inconclusive results (e.g., Strom \& Strom 1978 and references therein). We were prompted to investigate this when we noted that there are almost no very flat, high-luminosity galaxies in the 7SAM sample. In Figure $16 a$ we plot a scatter diagram of apparent ellipticity versus absolute magnitude, Velocities listed by the 7SAM were used to derive absolute magnitudes, and $H_{0}$ was assumed to be $50 \mathrm{~km} \mathrm{~s}^{-1} \mathrm{Mpc}^{-1}$. Some hint of an effect with absolute magnitude may be seen in this diagram, since the absence of flat bright galaxies is confirmed. It is also clear that the effect, whatever it is, is not very large. Next, we divided the sample into two equal-sized sets of intrinsically faint galaxies and luminous galaxies, and compared the ellipticity distribution within both sets. The result is shown in Figure $16 b$, and it does show a small difference, in that the bright ellipticals have a narrow distribution that peaks near $\epsilon=0.2$, while the faint galaxies have a flatter distribution extending to $\epsilon=0.6$. We repeated the procedure with the velocity dispersion as a discriminant, and show the results in Figures $16 c$ and $16 d$. The maximum difference in the cumulative histograms is about $21 \%$ and $25 \%$, respectively, for the two comparisons. The probability that both samples are drawn from the same parent distribution is 0.01 and 0.06 , respectively, according to the Kolmogorov-Smirnov test. When we used the 7SAM sample, we found similar fractions, which have higher significance because of the larger sample $(N=354)$.

What does this result mean? First, the difference may be (partly) due to the fact that we measure ellipticities at a constant isophote, and not at a constant structural radius such as
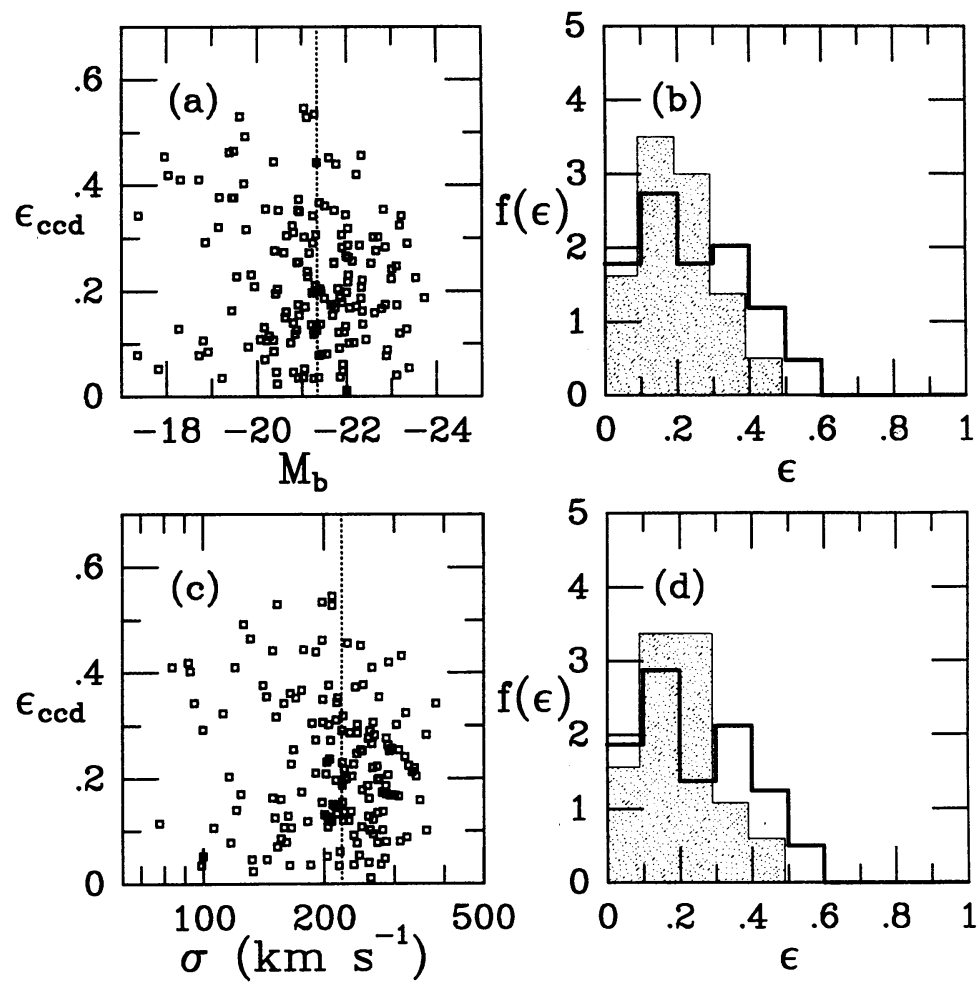

FIG. 16.-(a) Scatter diagram of ellipticity vs. absolute magnitude. The sample has been divided into two equal-sized parts, indicated by the line. The resulting

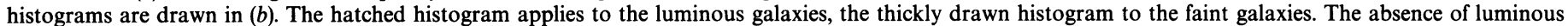

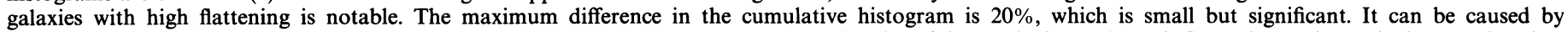

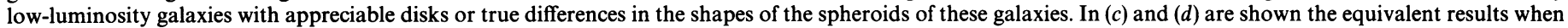
velocity dispersion $\sigma$ is used as a discriminant. 
the effective radius. If the ellipticity increases systematically with radius, then an effect like this may be expected. If we use ellipticities derived at an effective radius, the effect is less pronounced (the maximum difference in the cumulative histogram decreases to $0.13-0.18$ ), but it does not disappear. This is consistent with the results from Porter et al. (1991), who found that systematic gradients of the ellipticities of "normal" ellipticals are small. Second, we know that the rotational properties of ellipticals change as a function of mass. Faint ellipticals are generally flattened by their rotation, whereas bright ellipticals are generally flattened by velocity dispersion anisotropies (Davies et al. 1983). ${ }^{3}$ Thus it would be surprising if their flattening distributions were found to be identical. Third, the maximum difference in the cumulative histogram of ellipticities is only $20 \%$. The addition of a fraction of faint, flattened galaxies (ellipticals with disks?) at the $20 \%$ level could explain the whole effect. Various authors have argued that particular ellipticals have disks, either on grounds of photometry (e.g., Capaccioli 1987; Carter 1987; Bender et al. 1988) or on grounds of spectroscopy (e.g., Davies et al. 1983; Franx \& Illingworth 1988; Bender 1990). The statistical analysis by Rix \& White (1990) indicates that these disks may contribute significantly to the light, and could possibly influence the apparent ellipticity.

We do not think that the present data can resolve these issues. There appear to be small systematic differences between faint and bright ellipticals. These differences are either due to systematic differences in the flattenings of ellipsoids of different masses or due to systematic differences in the mass fraction of the disk with luminosity. This suggests that systematic differences could also occur in their kinematics and triaxiality, and may complicate the interpretation of the data.

\subsection{Distribution of Apparent Ellipticity and Misalignment}

Recently three major surveys of the kinematics of elliptical galaxies have been published. Davies \& Birkinshaw (1988, hereafter DB) have measured radial velocities along several position angles for 14 elliptical galaxies. Their galaxies were selected on the basis of their radio continuum morphology. Since these galaxies were not the extremely bright radio galaxies with disturbed appearances as found by Heckman et al. (1986), but regular ellipticals with possibly minor optical "features," we believe that these galaxies are relatively normal ellipticals. Franx, Illingworth, \& Heckman (1989b, hereafter FIH) studied a total of 22 galaxies, and presented radial velocities along the apparent major and minor axes. Their sample is biased toward round galaxies $(\epsilon<0.3)$. Most of the galaxies have an absolute magnitude in the interval $20<-M_{B}<22$. They were selected irrespective of radio properties or IR emission. Only two galaxies showed an indication of faint dust absorption, which is a somewhat lower fraction than generally observed (cf. Sadler \& Gerhard 1985). Jedrzejewski \& Schechter (1989, hereafter JS) observed 14 ellipticals along the major and minor axes. They selected their sample on the basis of ellipticity, ellipticity gradients, and position-angle gradients. Most of their galaxies have only small gradients. Unfortunately, this selection criterion might have introduced some bias, depending on the cause of the position-angle twists. If they are due to radial changes of the triaxiality parameter $T$, then the sample is biased toward galaxies which are seen at special viewing angles (those angles where the derivative of

\footnotetext{
${ }^{3}$ We ignore the very faint galaxies with $-M_{B} \leq 18$, which rotate slowly (e.g., Bender \& Nieto 1990; de Zeeuw \& Franx 1991).
}

position angle with respect to $T$ is small). If the kinematic misalignment is caused only by triaxiality, then these are angles at which one observes either small or large kinematic twists. The bias is not that strong if galaxies have kinematic misalignments caused by purely intrinsic misalignments (see $\S 3)$.

We note that neither ellipticity gradients nor position-angle twists were used to reject candidate galaxies in the first two surveys. Despite the normal caution one should always exercise when doing statistics on incomplete samples, we assume in the following that the combined sample is relatively free from gross selection effects. However, the sample is strongly biased toward round galaxies, and so we cannot use the histogram of observed ellipticities for these galaxies to test the models.

Scattered through the literature we found minor- and majoraxis data of varying quality on another 33 galaxies. Accurate two-dimensional surface photometry was available for only a small fraction of these galaxies. Only seven galaxies with goodquality observations along the major and minor axes and reliable surface photometry were found. All were unusual galaxies, e.g., NGC 5128 (Wilkinson et al. 1986), NGC 7097 (Caldwell, Kirshner, \& Richstone 1986), and NGC 3108 and NGC 5266 (Caldwell 1984). These galaxies were originally studied because of their well-known gas and dust features. We were reluctant to include these galaxies in our sample; not because they had observable gas and dust but because they were chosen as candidates for such studies as a result of the unique characteristics of their gas or dust features (often edge-on). Hence these galaxies are likely to have special orientations with respect to the line of sight, and are not suitable for statistical analysis. The galaxies that were published recently by Wagner et al. (1988) cannot be included because they were chosen for publication as a result of a striking kinematic property, namely, significant rotation along the minor axis.

The combination of the samples of DB, FIH, and JS yielded a sample of 44 galaxies. For six galaxies the misalignment angle is determined with accuracy worse than $25^{\circ}$. The misalignment angle becomes a meaningless number if the error is larger than $25^{\circ}$, and so we removed these galaxies from the sample. This may have introduced a small bias against those viewing angles which result in small observed rotational velocities (and thus relatively large uncertainties in those velocities), e.g., viewing directions almost parallel to the angular momentum.

We show histograms of misalignment angle, apparent ellipticities, and velocity amplitudes for these galaxies in Figure 17. The kinematic misalignment angles as determined by DB, FIH, and JS were used, with their error estimates. The ellipticities are recalculated from the CCD data base, and are ellipticities at $r=r_{e}$. An accurate error estimate for the kinematic misalignments remains a difficult task for this type of data. All three papers discuss this problem. We believe that the errors in the kinematic misalignments may be uncertain by a factor between 0.7 and 1.4. More details can be found in the papers where the samples are discussed. From the histogram of kinematic misalignment angles in Figure $17 a$ it is clear that there are galaxies with significant misalignments. We note that the distribution seems to peak around $0^{\circ}$ and at $90^{\circ}$, although the last peak contains only four galaxies and is therefore barely significant. We have also plotted the observed points in the $(\Psi, \epsilon)$-plane (Fig. 18). We see that the misalignment angle seems to be distributed independent of the observed ellipticity. This contrasts with many of the models which predict a strong 

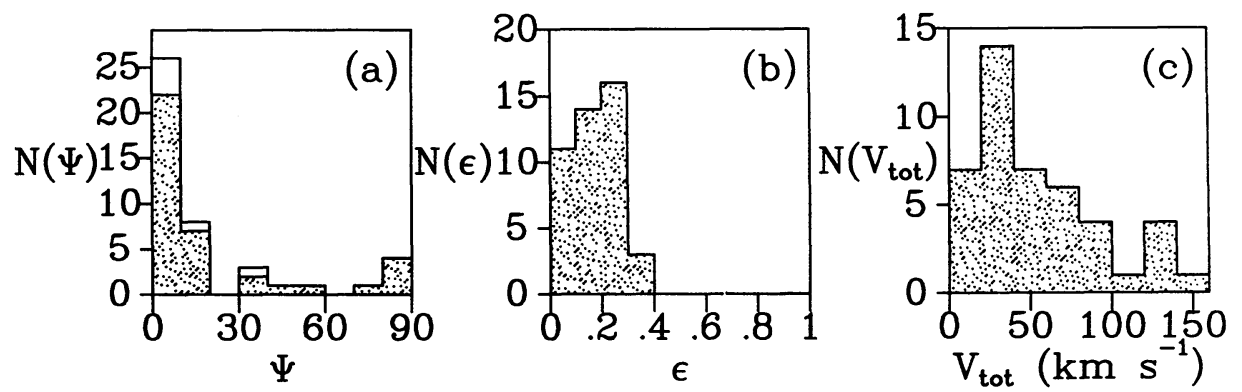

Fig. 17.- (a) Histogram of kinematic misalignment $\Psi$. The shaded histogram is for galaxies with an error in the misalignment smaller than $25^{\circ}$; the unshaded histogram includes all galaxies. In $(b)$ and $(c)$ are shown histograms of apparent ellipticity $\epsilon$ and rotation velocity amplitudes $v_{\text {tor }}$ for our sample. There is a relative absence of galaxies with $\epsilon \geq 0.3$.

anticorrelation between the ellipticity and the apparent misalignment (see $§ 3)$.

\section{MODELS OF INTRINSIC SHAPES AND INTRINSIC MISALIGNMENTS}

In this section we discuss the construction of distributions for the intrinsic flattenings, intrinsic misalignments of angular momenta, and intrinsic short axes that are in agreement with the data presented in the previous section. It is clear that such modeling is not unique, since we have three intrinsic parameters (intrinsic flattening $\epsilon_{1}$, triaxiality $T$, and intrinsic misalignment $\psi_{\text {int }}$ ) and two observed parameters (apparent ellipticity $\epsilon$ and apparent kinematic misalignment $\Psi$ ). However, certain properties of the apparent ellipticity and misalignment help us. The distribution of apparent ellipticities depends only on the distribution of $\epsilon_{1}$ and on the distribution of $T$. The distribution of the apparent misalignment depends only on the distribution of $T$ and the intrinsic misalignment $\psi_{\text {int }}$. Thus we decided to treat these as two separate inversion problems.

\subsection{Inversion of the Distribution of Apparent Ellipticities}

We inverted the histogram of CCD ellipticities (see $\S 4.1$ ). This problem is underconstrained, since two intrinsic parameters determine the distribution of one observable. A natural assumption that allowed the inversion of the apparent ellipticities was to assume that all galaxies have the same triaxiality $T$. We fitted a third-order polynomial to the observed (CCD data base) histogram of ellipticities, and inverted the resulting distribution with Lucy's (1974) method. This method guar-

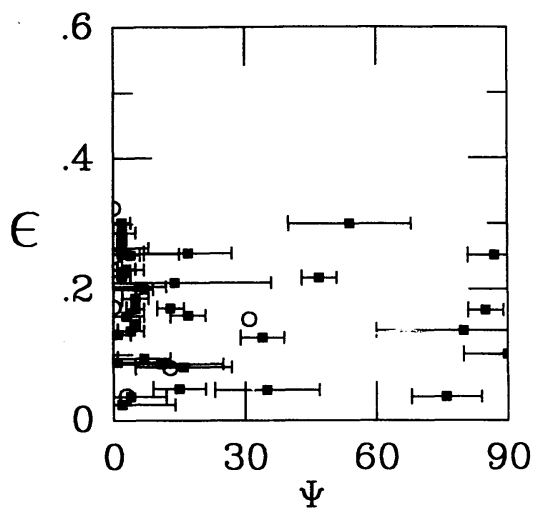

Fig. 18.-Observed galaxies in the $(\Psi, \epsilon)$-plane. Galaxies with errors in $\Psi$ larger than $25^{\circ}$ are indicated by open circles. It is striking that the distribution in $\Psi$ does not appear to correlate with $\epsilon$. antees smooth, positive solutions. Figure 19 shows the inverted distribution of $\epsilon_{1}$ for oblate models, intermediate triaxial models, and prolate models. All the distributions peak in the interval $0.3 \leq \epsilon_{1} \leq 0.5$. The oblate and prolate distributions go to zero at low ellipticity. The triaxial distribution shows a minimum at $\epsilon_{1}=0$. Note that the oblate distribution does not reproduce the dip in the histogram at low ellipticity; it predicts too many round galaxies. However, the difference is not statistically significant according to a Kolmogorov-Smirnov test (which does not have normal statistics, since the data are used to find the model solution). A similar result was found by Fasano \& Vio (1991). ${ }^{4}$ For the three cases of Figure 19, the mean intrinsic flattening is in the range $\left\langle\epsilon_{1}\right\rangle=0.3-0.36$.

Given the relatively small differences between the intrinsic distributions obtained with different values of $T$, we have not extended the analysis to other hypotheses concerning the shape. The distribution of apparent ellipticity is more sensitive to the distribution of intrinsic flattenings than the distribution of $T$, and hence many models with similar distributions of $\epsilon_{1}$ but different distributions of $T$ will fit the data.

\subsection{Inversion of the Distribution of Apparent Misalignment}

We next constructed models for the intrinsic misalignment $\psi_{\text {int }}$ and triaxiality $T$. There is again an ambiguity in the modeling, since two intrinsic parameters $\left(\psi_{\text {int }}\right.$ and $\left.T\right)$ determine the distribution of the observable parameter $(\Psi)$. Hence we constrained our solutions to lie on lines in the plane of intrinsic misalignment and triaxiality. We used the following procedure to obtain valid solutions: First we chose a line in the $\left(\psi_{\text {int }}, T\right)$ plane on which the solution lay, and defined 10 or 11 points $\left(\psi_{\text {int }, i}, T_{i}\right)$ on that line. We chose three different lines, and constructed three different classes of models, I, II, III, which are indicated in the $\left(\psi_{\text {int }}, T\right)$-plane in Figure 20. Models of class I have no intrinsic misalignment; all the apparent misalignment is due to the triaxiality. Models of class II have no triaxiality, and all the apparent misalignment is due to intrinsic misalignment. These two classes are extremes, and thus they can be contrasted with each other. Models of class III have nonzero triaxiality and intrinsic misalignment, and these parameters are linearly related.

Next, for each of the points on the line given by a particular class of model we inverted the distribution of apparent ellipticities for the triaxiality $T$ of that point. Then we calculated the distribution $p\left(\epsilon, \Psi \mid \psi_{\mathrm{int}, i}, T_{i}\right)$ for that distribution of $\epsilon_{1}$ and

\footnotetext{
${ }^{4}$ Fasano \& Vio (1991) claimed that the difference is significant at the $3 \sigma$ level, but they base this on the $3 \sigma$ deviation of the lowest ellipticity bin. The probability that 1 bin out of 13 differs by more than $3 \sigma$ is not negligible, however.
} 


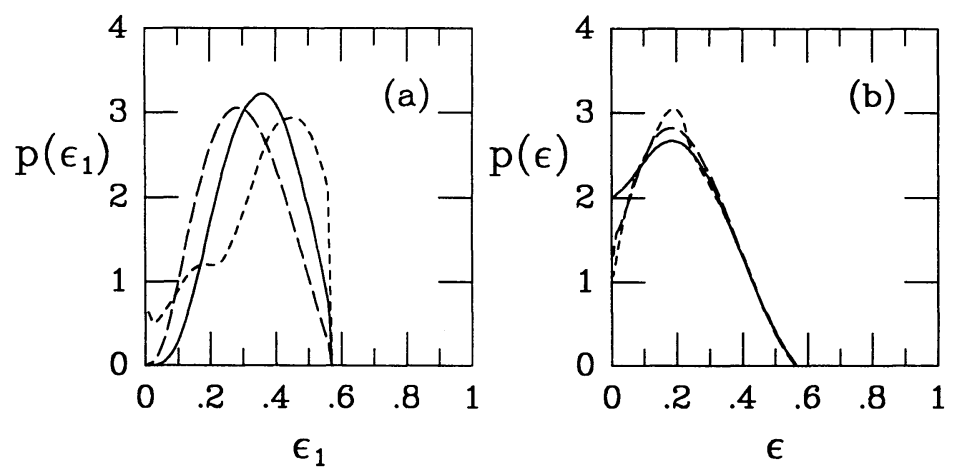

FIG. 19.- (a) Inverted distribution of intrinsic flattenings $p\left(\epsilon_{1}\right)$ for three different values of the triaxiality parameter $T$. The continuous line is for $T=0$ (oblate), the short-dashed line for $T=0.5$, and the long-dashed line for $T=1$ (prolate). All models have very few intrinsically round galaxies. In $(b)$ is shown the distribution of apparent ellipticities for these models (cf. Fig. 14 for the data). The oblate model is not fully able to reproduce the observed minimum at $\epsilon=0$.

the specific values of $\psi_{\mathrm{int}, i}$ and $T_{i}$ of the point. A wide range of models can be constructed by varying the weights $w_{i}$ of the "building blocks" $p_{i}(\epsilon, \Psi)$. The resulting probability distribution $p(\epsilon, \Psi)$ is given by

$$
p(\epsilon, \Psi)=\sum_{i} w_{i} p_{i}\left(\epsilon, \Psi \mid \psi_{\mathrm{int}, i}, T_{i}\right)
$$

and is easily evaluated. The procedure guarantees that this probability distribution reproduces the observed distribution in apparent ellipticity. We next constructed distributions in two different ways:

1. We produced smooth models with Lucy's (1974) algorithm. The probability distributions $p_{i}(\epsilon, \Psi)$ were averaged over the interval $0 \leq \epsilon \leq 0.3$, reflecting the interval in ellipticities in which the galaxies lie. The resulting distributions were smoothed in $\Psi$ to represent the average measurement error of 6:6. Afterward, they were divided in bins of $10^{\circ}$. Finally, the data histogram was inverted with Lucy's algorithm to obtain a smooth distribution of weights $w_{i}$.

2. We chose to explore the solutions space through linear

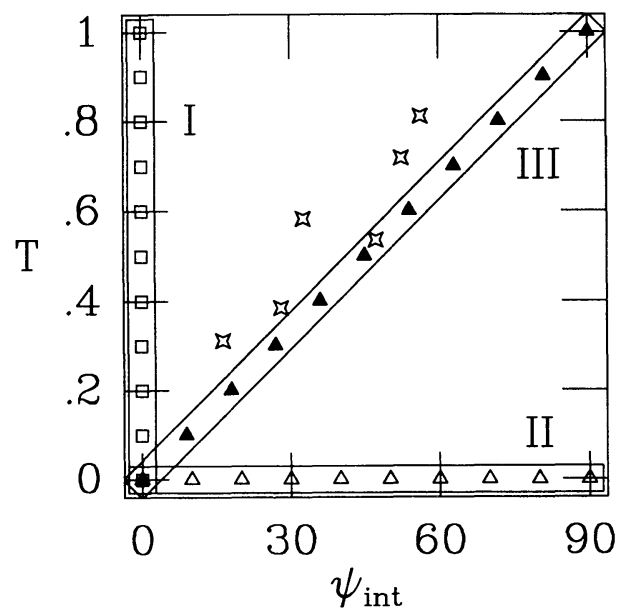

FIG. 20.-Plane of intrinsic misalignment $\psi_{\text {int }}$ and triaxiality $T$. The three classes of solutions I, II, and III are indicated. Class I consists of triaxial galaxies without intrinsic misalignments, class II of oblate galaxies with intrinsic misalignments, and class III of triaxial galaxies with intrinsic misalignments. The squares and triangles indicate the models which are used to reproduce the observed misalignments for each class of solution. The stars are the dynamical models by Statler (1987), his "Lucy" models. The angular momenta are assumed to have their maximum values, both along the $x$-axis and along the $z$-axis. Solution III lies near to these points. programming and systematic searches. For that we needed a goodness-of-fit estimator, that allowed us to reject or accept models. We used the adapted Kolmogorov-Smirnov test devised by Binney (1985). For a given model, the cumulative probabilities $P\left(\Psi>\Psi_{\text {gal }} \mid \epsilon_{\mathrm{gal}}\right)$ are evaluated for each galaxy. If the model is the true model, then these probabilities are randomly distributed between 0 and 1 . This was tested by means of the Kolmogorov-Smirnov test, which is based on the maximum difference $D_{\max }$ between the observed and model distributions of cumulative probabilities (a linearly rising function). We rejected models at the $95 \%$ confidence level (i.e., the probability of falsely rejecting the true model was smaller than $5 \%$ ).

In principle, a full exploration of the solution space is a complex mathematical problem, and it is not attempted here. Instead, we first constructed models by using an approximate technique: if the measurement errors in $\Psi$ are identical for all galaxies, and if the dependence of $p(\epsilon, \Psi)$ on $\epsilon$ can be ignored, then the adapted Kolmogorov-Smirnov test reduces to a regular Kolmogorov-Smirnov test which can be expressed as a set of linear constraints on the weights $w_{i}$. We name this reduced test the limited Kolmogorov-Smirnov test. Next we used the linear programming (LP) technique to find a wide range of solutions. We adapted the code from Press et al. (1986). The LP technique allows us maximize a function (the "cost function"). We optimized linear combinations of $\langle q\rangle$ and $\left\langle q^{2}\right\rangle$, where $q$ is the changing parameter along the sequence of the model "building blocks" $p_{i}(\epsilon, \Psi)$; thus $q=T$ for solution classes I and III, and $q=\psi_{\text {int }}$ for solution class II. A systematic survey of all linear combinations of $\langle q\rangle$ and $\left\langle q^{2}\right\rangle$ gives us the total allowed space in the $\left(\langle q\rangle,\left\langle q^{2}\right\rangle\right)$-plane. The models generated by this technique are of an extreme nature. Many of them have only one or two nonzero weights $w_{i}$. They have the special property that a linear combination of valid models will again produce a valid model (according to the limited Kolmogorov-Smirnov test). When we tested the models produced by the LP technique with the original adapted Kolmogorov-Smirnov test, we found that some of them were ruled out at the $95 \%$ confidence limit; apparently the differences in the measurement errors and the dependence on $\epsilon$ cannot be ignored.

Based on this experience, we decided to build "synthetic" models, and tested these models with the adapted Kolmogorov-Smirnov test. We tried three types of models: (1) Models with the weights $w_{i}$ a linear function of parameter $q$; the slope was varied extensively, and any resulting negative 
weights were substituted by zeros. (2) Models with the weights a Gaussian function of $q$; the mean and dispersion around the mean were varied. (3) Models with only two nonzero weights (" two-peaked models"). All possible combinations were tested, with steps of 0.01 in the nonzero weights. Our experience with the LP fitting showed that the last type of model explores the solution space quite adequately, although we have no guarantee that it gives a full coverage. Below we discuss the resulting models for each class of solutions.

\subsubsection{Class I: Models with Perfect Intrinsic Alignment}

We first analyzed models for which the apparent misalignments are entirely due to projection effects. The angular momentum was assumed to be parallel to the intrinsic short axis, or $\psi_{\text {int }}=0$.

We evaluated 11 models along the line of $\psi_{\text {int }}=0$, with $T=i / 10, i=0,10$. We used Lucy's method to construct a smooth, well-fitting model. We call this solution Ia; it is shown in Figure 22a below. It is nonzero from $T=0$ to $T=1$, and thus covers all shapes from oblate to prolate. The mean triaxiality is 0.41 . The adapted Kolmogorov-Smirnov test indicates that it is a good fit to the data.

We next explored the solution space more systematically through our synthetic models described above. We show the covered range in the $(\langle T\rangle, \sigma(T))$-space in Figure 21. We derive limits on the mean triaxiality $0.14 \leq\langle T\rangle \leq 0.7$, and on the dispersion in triaxiality $0<\sigma(T) \leq 0.48$. Some models with zero dispersion in triaxiality (i.e., a single delta function) are allowed by the adapted Kolmogorov-Smirnov test, but they are not a good fit, since the galaxies with observed misalign-

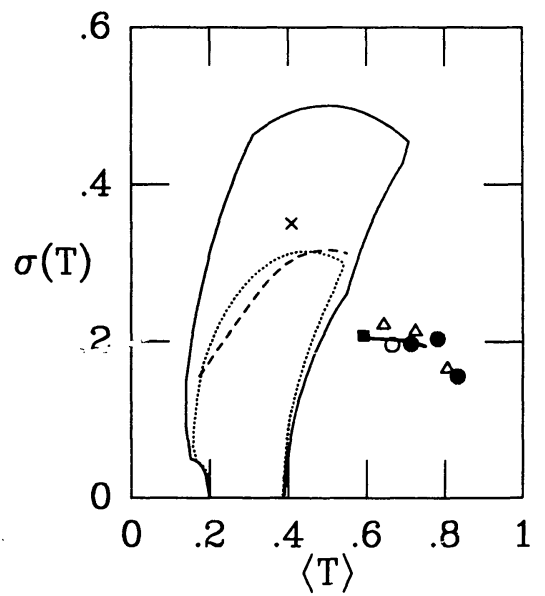

Fig. 21.- Solution space of mean triaxiality $\langle T\rangle$ and dispersion in triaxiality $\sigma(T)$ for models with perfect intrinsic misalignment $\left(\psi_{\text {int }}=0\right)$. The large region bounded by the continuous line is the region of acceptable solutions with two delta functions in triaxiality (the "two-peaked" models). The region bounded by the dotted line is the region of acceptable models with a Gaussian distribution of $T$. The dashed line represents acceptable solutions with weights that are a linear function of $T$. The cross is the Lucy solution Ia. The points are predictions for the shapes of dark halos from numerical simulations of hierarchical formation scenarios. The square is for the distribution predicted by Frenk et al. (1988); the triangles and filled circles are for distributions by Dubinski \& Carlberg (1991) and Warren et al. (1991a, b). The three different symbols are for three different radii within the halos, with the central shapes being the most prolate. The open circle is the distribution of shapes predicted by White \& Ostriker (1990) for halos formed on shells around voids. The line through the points is the analytical prediction of shapes of halos by Bardeen et al. (1986). All of these predictions can be ruled out to apply to the luminous parts of ellipticals. ments $\Psi \geq 45^{\circ}$ and $\epsilon \geq 0.2$ are forbidden by these models. The presence of these galaxies require nonzero weights $w_{i}$ with $T_{i} \geq 0.5$. However, an infinitesimal contribution from these components will allow the relatively flattened, misaligned galaxies, and thus we only require $\sigma(T)$ to be nonzero. The high mean triaxialities occur only for models with large $\sigma(T)$. These are models with two widely separated peaks in $T$. We show an example in Figure $22 a$ (solution Ib). It has a mean triaxiality of 0.5 and has only oblate and prolate shapes. It clearly provides a good fit to the observed histogram, not much better or worse than the Lucy model. These two extremes illustrate well the ambiguity of the problem: a wide range of solutions is possible.

\subsubsection{Class II: Oblate Models with Intrinsic Misalignments}

Next we assumed that the apparent misalignments were due to intrinsic misalignments. The shapes of the galaxies were assumed to be nearly oblate $(T \approx 0)$, so that we could use the results of $\S 3$ for oblate models. The results should be contrasted with those of the preceding subsection, where we assumed that the apparent misalignments are mainly due to projection effects of the triaxial shapes of galaxies. We chose 10 points to lie along the line $T=0$ with $\psi_{\text {int }}=i \times 10^{\circ}, i=0,9$. The distribution of $\epsilon_{1}$ is that for oblate galaxies $(T=0)$. We have again constructed the probability distributions $p(\epsilon, \Psi)$ for all points.

The Lucy solution is shown in Figure $22 c$ (solution IIa). It has two peaks at $\psi_{\mathrm{int}}=0$ and $\psi_{\mathrm{int}}=90^{\circ}$ and is nonzero in between. It has a mean intrinsic misalignment of $24^{\circ}$. It is a good fit to the data by the Kolmogorov-Smirnov test. We again explored the solution space with our synthetic solutions. We found various models with linear, Gaussian, or two-peaked distributions that fitted the data satisfactorily. Again, the presence of flattened, misaligned galaxies requires a small contribution from components with $\psi_{\text {int }}>40^{\circ}$, but this is not a strong constraint, as an infinitesimal contribution is sufficient. The range in mean intrinsic misalignment is from $4^{\circ}$ to $45^{\circ}$. The maximum is reached for a model with two peaks, at $0^{\circ}$ and $90^{\circ}$, with equal contributions. Of interest is the minimum required fraction of galaxies with small intrinsic misalignments. We obtain the following numbers from our modeling: $f\left(\psi_{\text {int }}<5^{\circ}\right) \geq 0.05, f\left(\psi_{\text {int }}<15^{\circ}\right) \geq 0.35, f\left(\psi_{\text {int }}<25^{\circ}\right) \geq 0.40$. Again, we stress that we have no guarantee that these numbers are the absolute minima allowed by the adapted KolmogorovSmirnov test, but we believe that they are a good approximation. The maximum fraction of galaxies with misalignments greater than $45^{\circ}$ is 0.5 (e.g., our maximum $\left\langle\psi_{\text {int }}\right\rangle$ model). We show an example of one of the extreme models in Figure 22c (solution IIb). This is a model with two peaks, at $\psi_{\text {int }}=0^{\circ}$ and $90^{\circ}$, with relative weights of 0.6 and 0.4 . It fits the data well according to the adapted Kolmogorov-Smirnov test $\left[p\left(D_{\max }>D_{\max , \text { obs }}\right)=0.45\right]$.

\subsubsection{Class III : Triaxial Models with Intrinsic Misalignments}

Third, we assumed that all galaxies lie along a line in the $\left(\psi_{\text {int }}, T\right)$-plane, passing through the points $\left(\psi_{\text {int }}, T\right)=(0,0)$ and $\left(1,90^{\circ}\right)$. The choice of this line was based on the dynamical models produced by Statler (1987). He calculated the maximum angular momenta along the long axis and the short axis for a variety of models. We analyzed his "Lucy" models, which have smooth phase-space distribution functions. His self-consistent models specify the occupation numbers of the tube orbits, but they do not specify whether all stars rotate in the same sense around the rotation axis within one tube orbit, 


$$
\begin{aligned}
& \text { I } \psi_{\text {int }}=0 \\
& \text { Ia } \\
& \text { OIb }
\end{aligned}
$$
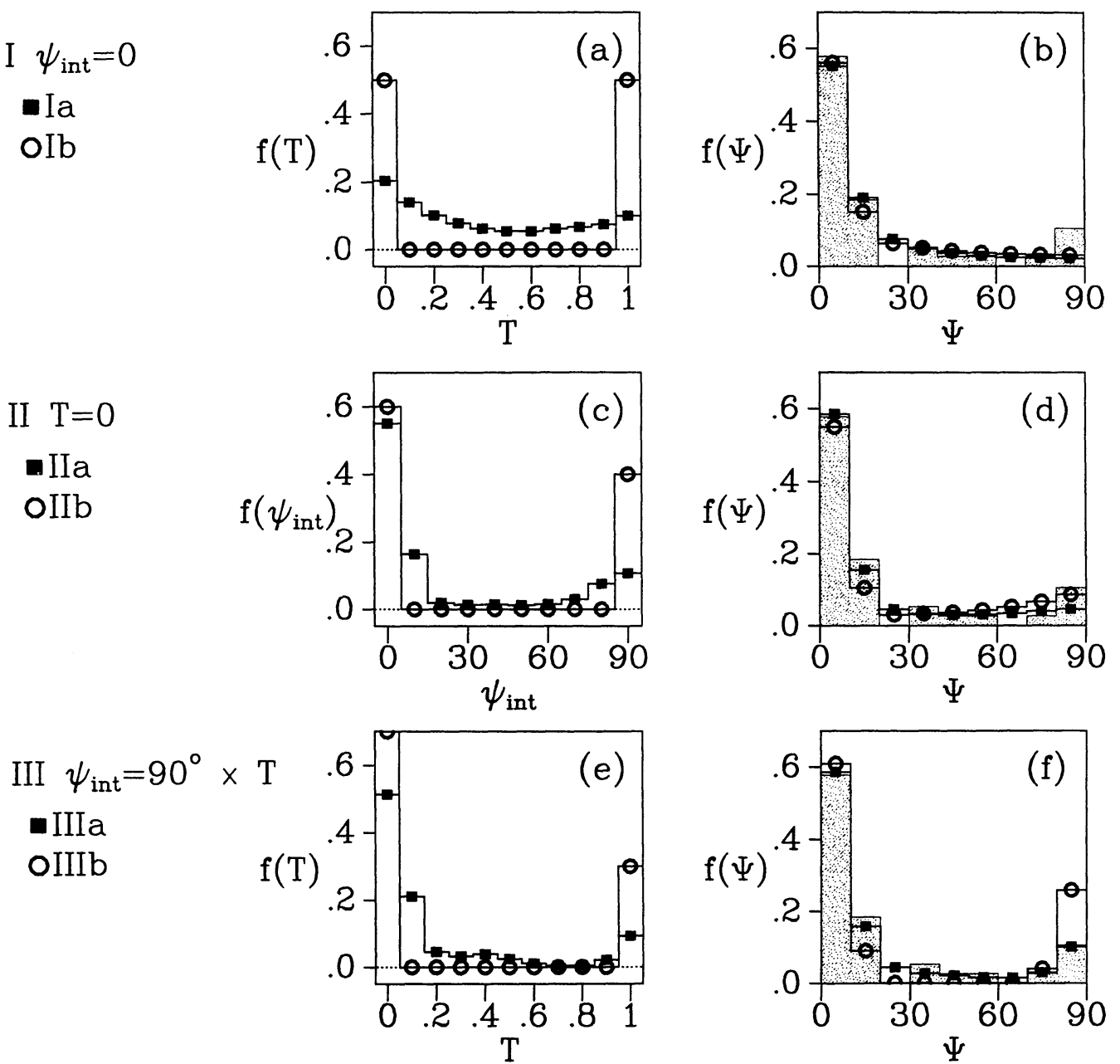

FIG. 22.- Solutions for the intrinsic shapes and intrinsic misalignments of elliptical galaxies. (a) Solutions of class I, i.e., triaxial galaxies without intrinsic

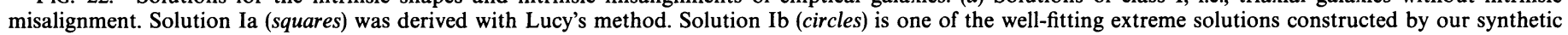

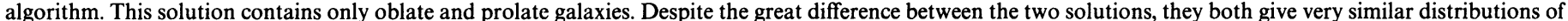

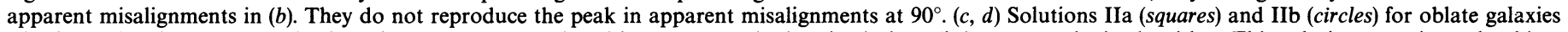

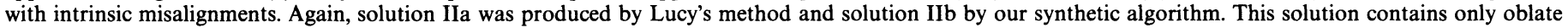

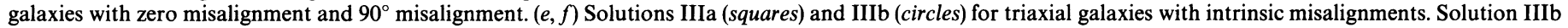
provides a satisfactory fit according to the Kolmogorov-Smirnov test, and consists only of oblate short axis rotators and prolate long axis rotators.

or within one class of tube orbits rotating around the axis. We assumed that all the stars rotating around the $x$-axis are rotating in the same sense, and that all the stars rotating around the $z$-axis are rotating in the same sense. Now the maximum angular momenta along the $x$-axis and the $z$-axis specify the full angular momentum, and the intrinsic misalignment and the triaxiality can be evaluated for these models rather easily. We have shown the points for all Statler's "Lucy " models with $\epsilon_{1}<0.5$ in Figure 20, and we can see that these points lie reasonably near the indicated diagonal. We chose points along the diagonal with $T=i / 10, \psi_{\text {int }}=i \times 9^{\circ}, i=0,10$. The histogram of apparent ellipticities was inverted for all these points, and the probability distributions $p(\epsilon, \Psi)$ were evaluated.

The Lucy solution is shown in Figure 22e. Solution IIIa is positive for all points and has two distinct peaks, at $\psi_{\text {int }}=0^{\circ}$ and $\psi_{\text {int }}=90^{\circ}$. The solution reproduces the peak at apparent misalignment of $90^{\circ}$ very well (better than the previous Lucy solutions). The mean triaxiality of the model is 0.19 , and the mean intrinsic misalignment is $17^{\circ}$. Note that the mean triaxiality is more than a factor of 2 lower than the triaxiality of solution Ia, but that the mean intrinsic misalignment is only $20 \%$ lower than that of solution IIa. Apparently, the intrinsic misalignment dominates over the triaxiality in producing the apparent misalignment for this class of model. Again, we explored the solution space more thoroughly using our synthetic models. We found that the mean triaxiality varied between 0.045 and 0.35 for acceptable models. The highest mean triaxiality was reached for a model with two peaks, at $T=0$ and $T=1$, with relative fractions of 0.65 and 0.35 , respectively. Note that this is a combination of oblate short axis rotators and prolate long axis rotators. It predicts galaxies to have true apparent misalignments of $0^{\circ}$ or $90^{\circ}$, and nothing in between. Thus the galaxies with intermediate misalignments are explained as galaxies with large measurement errors. We do not think that this conclusion is correct; it emphasizes one of the problems related to the Kolmogorov-Smirnov test: even though a galaxy might lie in a region for which $p(\epsilon, \Psi)$ is zero, the model will not be rejected because the cumulative probabilities are used to test the model. Again, this problem can be fixed by allowing for an infinitesimally small contribution of 
galaxies with intermediate misalignments and triaxialities. We cannot put useful constraints on the minimum fraction of such galaxies. In Figure $22 e$ we show a model with nonzero weights at $T=0$ and $T=1$. The relative contributions are 0.7 and 0.3 , respectively. This model was chosen because it is extreme, but it gives a reasonable fit according to the Kolmogorov-Smirnov test $\left[p\left(D_{\max }>D_{\max , \text { obs }}\right)=0.13\right]$.

\subsubsection{Mixed Models}

We have argued before that at least some fraction of galaxies are long axis rotators (e.g., NGC 4365, NGC 4406). This stimulated us to consider models for which the galaxies are either triaxial with no intrinsic misalignment or have $\psi_{\text {int }}=90^{\circ}$. Hence these models are combinations of short axis rotators and long axis rotators. We considered two special cases: one for which all long axis rotators are prolate $(T=1)$, and one for which the long axis rotators are oblate $(T=0)$. Real long axis rotators cannot be oblate, because oblate galaxies have only rotation around their short axes. Hence this case should be considered as the hypothetical limiting case (i.e., small $T$ ). We cannot give firm lower limits to the fraction of long axis rotators. This follows from the fact that we can fit the data sufficiently well by only short axis rotators (models of class I). We can put some upper limits on the long axis rotators. We explored the solution space with our "two-peaked" models, and we found that the maximum fraction of prolate long axis rotators is $35 \%$, and of oblate long axis rotators is $50 \%$. The best-fitting "two-peaked" model with prolate long axis rotators had a fraction of 0.90 galaxies at $T=0.3$ and a fraction of 0.10 of prolate long axis rotators. Curiously enough, this model was the best-fitting model of all models that we constructed (of all classes). We should not attach too much value to that, because we made no systematic effort to find the best-fitting models. The best-fitting "two-peaked" model with oblate long axis rotators had a fraction of galaxies with $T=0.3$ of 0.85 , and a fraction of 0.15 of oblate long axis rotators.

\subsubsection{General Characteristics of the Solutions}

In summary, we have produced solutions on three lines in the $\left(\psi_{\text {int }}, T\right)$-plane. There is no reason to believe that real galaxies are constrained to lie on a line in a plane, and so it should be possible to construct much more general solutions. Given the degeneracy of the mathematical problem, we did not attempt to produce such solutions. The previous solutions do suggest some important constraints on the models: (1) all the solutions have a sizable fraction of galaxies with intrinsic misalignments smaller than $15^{\circ}$ (at least $35 \%$ ). This is needed to reproduce the strong peak at observed misalignments of $0^{\circ}-10^{\circ}$. (2) The small peak of galaxies near misalignments of $90^{\circ}$ can be reproduced quite well with a small fraction $(10 \%-$ $20 \%$ ) of galaxies that rotate round their long axes. These galaxies can be near to oblate or prolate. The current data do not allow us to put strong constraints on their shapes. (3) All solutions satisfy the following constraint on the mean triaxiality and mean intrinsic misalignments: $\langle T\rangle \leq 0.70$ and $\left\langle\psi_{\text {int }}\right\rangle \leq$ $45^{\circ}$. These large values of $\langle T\rangle$ and $\left\langle\psi_{\text {int }}\right\rangle$ can only be reached for models with large dispersions in these parameters. The distributions have to have two separated peaks to reach these values. We cannot put interesting lower limits on $\langle T\rangle$ and $\left\langle\psi_{\text {int }}\right\rangle$. These results can be improved by extending the data set to large ellipticities. A larger data set would also help to establish the fraction of galaxies with large intrinsic misalignments.

\section{PREDICTIONS FROM HIERARCHICAL FORMATION MODELS}

The formulation of the cold dark matter hypothesis (Peebles 1982; Blumethal et al. 1984) to explain the formation of the structure in the current universe has stimulated intensive research into the detailed properties of halos in this model. Two types of predictions have been made about the shapes of halos. First, Doroshkevich (1970) and Bardeen et al. (1986, thereafter BBKS) have given predictions for the shapes of peaks in Gaussian random fields. These predictions should be regarded as predictions of initial conditions, before the structures collapsed and became nonlinear. BBKS give very general formulae which can be applied easily to hierarchical models with different power spectra. Second, numerical simulations of the collapse of structure have been analyzed to yield distributions of intrinsic shapes of halos (e.g., Frenk et al. 1988). Most of these simulations included only the dissipationless dark matter component. The similarity between the dynamics of halos and elliptical galaxies may be taken as an indication that they have similar shapes. However, there is no compelling reason that this should be so. Nevertheless, the comparison between the halo predictions and the constraints provided by our data may give new constraints on the formation mechanism of the luminous parts.

\subsection{Analytical Predictions for the Shapes of Peaks}

BBKS derived the intrinsic shapes of the peaks in their Gaussian random fields by evaluating the probability distribution of the spatial second derivatives at each peak. This derivation requires the random field to be continuous and differentiable. Random fields with power spectra $P(k)=k^{n}$, where $k$ is the frequency, are generally not differentiable. These fields are usually smoothed by a Gaussian on the scale of the object (here galaxy halos) to obtain a useful result. BBKS showed that the shape distribution is a function only of the spatial size of the peak, expressed by their parameter $x$. The shape is not directly related to the height of the peak. The size parameter is correlated with the height of the peak in a statistical sense, however. For power spectra with indices between 0 and -2.5 , the parameter $x$ generally lies between 1 and 6 , for peak heights between 1 and 6 times the standard deviation of the field. We transformed the probability distribution for the shapes given by BBKS into the probability distribution of $\epsilon_{1}$ and $T$. For the derivation see Appendix B. In Figure $23 a$ we show the distribution of triaxiality for various values of $x$. For all values of $x$ in the relevant range, the models predict a peak at high $T$, and the mean triaxiality varies between 0.6 and 0.75 for $x=6$ and $x=1$, respectively. This shows that the initial conditions for halos can be thought to be very triaxial-prolate. We have drawn the line of $\langle T\rangle$ and $\sigma(T)$ in Figure 21. Clearly, it lies outside the range of our acceptable synthetic solutions. When we apply the adapted Kolmogorov-Smirnov test to these models (see $\S 5$ ), we find that we can rule out these distributions at the $0.995 \%$ confidence level. Thus ellipticals do not have the typical triaxialities predicted for halos in their initial stages of collapse.

\subsection{Numerical Results of the Shapes of Peaks}

The collapse of the halos will certainly alter their shapes. The direction of the effect is not very predictable in the nonlinear regime. In the linear regime, structures will become flatter, (i.e., $\epsilon_{1}$ increases), but the triaxiality does not necessarily change by a large factor. Many authors have performed 

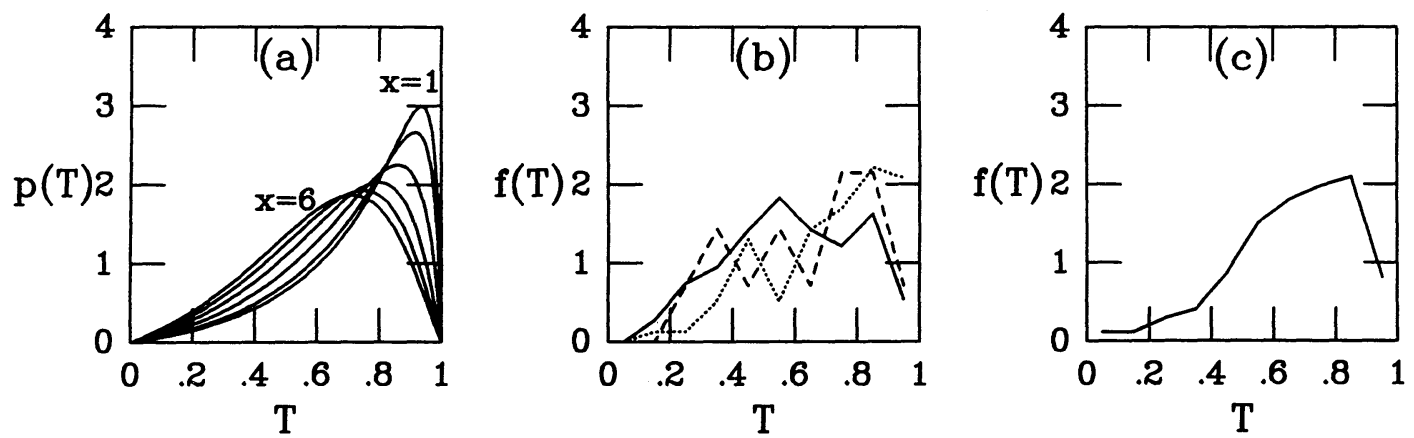

Fig. 23.- (a) Predicted distribution of the triaxiality of peaks in Gaussian random fields. These distributions are thought to be typical of the initial conditions before collapse. The distributions depend on the parameter $x$, which is a measure of the size of the peak. This parameter was varied between 1 and 6 in steps of 1 , and covers the relevant range for most power spectra. (b) Distribution of triaxiality of collapsed halos in hierarchical formation scenarios. The continuous line is based on simulations by Frenk et al. (1988), the dotted line on simulations by Warren et al. (1991b), and the dashed line on simulations by Dubinski \& Carlberg (1991). We show the least prolate-triaxial distributions from the last two authors. All three distributions are inconsistent with the data on the luminous parts of ellipticals. (c) Distribution of triaxiality taken from White \& Ostriker (1990), for halos forming on shells around voids. This distribution is comparable to the others and inconsistent with the data on the luminous parts of ellipticals.

numerical simulations of this process, and have measured the shapes of the resulting halos (Frenk et al. 1988; Dubinski \& Carlberg 1991; Warren et al. 1991a, b). Generally, they have found that the halo shapes were more flattened than elliptical galaxies (and, of course, much larger!). We determined the distribution of triaxialities of the halos in the three sets of simulations, and we present these in Figure $23 b$. It is remarkable that the distributions look rather similar, despite large differences in resolution and particles per halo between the different authors. This gives us some confidence in the result. We have plotted the mean triaxialities and dispersions in triaxiality in Figure 21. They overlap well with the BBKS predictions, but they extend to more triaxial-prolate shapes. All of these shape distributions can be ruled out to apply to the luminous parts of ellipticals at the $99.3 \%$ confidence level or better. This analysis assumes that the angular momentum is perfectly aligned with the intrinsic short axis, which is probably not the case for structures formed in this way. If we were to include the intrinsic misalignments, the discrepancy with the data would only become worse!

There may be several reasons for the difference between the halo shapes and the luminous parts of ellipticals. First of all, the simulations may still lack the resolution needed to get the proper shapes in the central parts of the halos. Miralda-Escudé \& Schwarzschild (1989) showed that the regular box orbits which sustain the shapes of triaxial galaxies become unstable in scaleless potentials or potentials with small core radii. This effect may be relevant for dark halos. The numerical dark matter simulations may not have enough radial resolution to find this effect. Hence, with much higher resolution, the halos may become more axisymmetric. Second, it is possible that significant gradients in triaxiality occur in these halos. It is remarkable to note, however, that both Dubinski \& Carlberg and Warren et al. find gradients in the triaxialities of their halos, with the inner parts being more prolate (which exacerbates the problem). Third, our data concern only the luminous parts of galaxies, and these may very well have shapes different from those of the halos. Ellipticals may have formed from "galaxies" with significant substructure (e.g., protogalaxies with bars and disks). Thus, the luminous parts may have shapes different from those of the dark halos caused by the (dynamically cold) structure of the luminous material prior to the last "merger event" in which the galaxy was assembled. Finally, the effects of dissipation and hydrodynamics have not been included in most simulations. Gas may help to make galaxies more axisymmetric because it tends to settle in (nearly) circular disks. Thus any gas settling may help to make the shapes of the luminous part more axisymmetric, and it might even help to make the whole halo more axisymmetric. It is noteworthy that exactly this effect was found by Katz \& Gunn (1991) in their collapse simulations. If this process is the most relevant, then it may help to put constraints on the fraction of mass in gas at the last round of major "mergers" which formed the present galaxies. It would be useful to investigate how much mass has to be added on the stable gas orbits to influence the shape of a triaxial galaxy significantly.

It is also possible, of course, that galaxies formed in a way quite different from that in the hierarchical formation scenarios. We are unaware of detailed predictions of the shapes of halos or galaxies in alternative scenarios, except for the work of White \& Ostriker (1990). These authors analyzed the halos formed on expanding shells of dark matter around voids. They found that their halos are more spherical than the halos produced in hierarchical scenarios. We determined the distribution of triaxialities, and plotted this in Figure 23c. Remarkably, the distribution is very similar to the distributions for hierarchical scenarios. Such a distribution is likewise ruled out to apply to the shapes of ellipticals. The same uncertainties as discussed for the hierarchical models apply to this type of model. Summarizing the above results, it might be hard to distinguish between formation scenarios on the basis of the shapes of ellipticals, but it may be possible to find interesting constraints on the formation of their luminous parts (in addition to constraints from their relative sizes, densities, flattenings, angular momenta, $M / L$ ratios, etc.).

\section{CONCLUSION}

We have constructed a variety of models which are able to reproduce the observed distribution of apparent ellipticities and apparent kinematic misalignments. The models are constrained by two different sets of data. The first is the distribution of observed ellipticities. These were drawn from a large data set of accurate CCD images that has been reported in the literature over the last few years. The new data show a relative paucity of nearly round galaxies, in agreement with other recent analyses. The distribution differs markedly from the older distribution of ellipticities obtained from the RC2. This is presumably due to the inhomogeneous data quality and non- 
quantitative determinations used for the older photographic results. The second data set is a compilation of apparent kinematic misalignments from the recent literature, using those three surveys of the rotation along the minor and major axes of ellipticals that are statistically sound.

The distribution of apparent ellipticities for high-luminosity ellipticals and low-luminosity ellipticals are somewhat different. There is a relative absence of highly flattened, highluminosity galaxies. This may reflect a true difference in the flattenings of the ellipsoids, since the shapes of luminous galaxies appear generally to result from velocity dispersion anisotropies, while the shapes of faint galaxies result from rotation. Alternatively, contamination by galaxies with significant disks in the faint sample could cause a similar effect. At least $20 \%$ of faint ellipticals would need to have such disks to explain the difference that is seen.

The determination of the intrinsic shape and intrinsic misalignment of the angular momentum from the intrinsic short axis is degenerate. Three intrinsic parameters (ellipticity, triaxiality, and intrinsic misalignment) are constrained by two observable parameters (apparent ellipticity and apparent kinematic misalignment). The problem simplifies somewhat by using the special property that the apparent kinematical misalignment depends only on the intrinsic misalignment and triaxiality, and so the ellipticity drops out. Hence we regarded the two problems of finding the intrinsic flattening and finding the intrinsic triaxiality and misalignment as separate ones. The inversion of the apparent ellipticities depends only weakly on the assumed triaxiality of galaxies. For all triaxialities, the distribution of intrinsic flattenings shows a peak at an ellipticity between 0.3 and 0.45 , and declines to a low value at $\epsilon_{1} \equiv 1$ $-c / a=0$. For oblate and prolate models the inverted intrinsic distribution has no round galaxies, and for triaxial systems the fraction of galaxies with $\epsilon<0.1$ is small (on the order of $7 \%$ ).

Many models could be constructed to reproduce the histogram of apparent kinematic misalignments. Models with all galaxies triaxial and well-aligned angular momenta, models with all galaxies oblate and nonaligned angular momenta, and more general models (triaxial with intrinsic misalignments) worked equally well. We argue from the presence of kinematical subsystems in the cores of NGC 4365 and NGC 4406 that these galaxies have angular momenta oriented close to the long axis. Hence, some galaxies are likely to have intrinsically misaligned angular momenta, and models which do not allow for such misalignments are too limited. The results from models which include galaxies with large intrinsic misalign- ments suggest that $10 \%-20 \%$ is a reasonable estimate of the fraction with such intrinsic misalignments. The current data cannot constrain their triaxiality; they can range from oblate to prolate. The solutions that we produce can be either relatively smooth or very peaked. All solutions give acceptable fits to the data, as tested by an adapted version of the Kolmogorov-Smirnov test. All the solutions require at least $35 \%$ of galaxies to have misalignments smaller than $15^{\circ}$. It is remarkable that the momenta are so well aligned with the intrinsic short axis, since this is not required for triaxial galaxies. The maximum mean triaxiality $T$ that is allowed is 0.7 , on a scale where $T=0$ for oblate and $T=1$ for prolate shapes. The maximum mean misalignment is $45^{\circ}$. It will be interesting to see whether one formation mechanism can account for both the large fraction of galaxies with very small intrinsic misalignments and the small fraction of galaxies with large intrinsic misalignments.

The resulting triaxiality and misalignment distributions can be compared with the predictions from theories of hierarchical formation of galaxies. Analysis of the shapes of the density peaks before collapse predicts highly triaxial-prolate structures. Numerical simulations have also produced halos that are comparably triaxial-prolate. These shapes are systematically more triaxial-prolate than the luminous parts of ellipticals. This comparison should not be considered to be a reflection upon the hierarchical formation picture. First, the simulations may not yet have the required resolution. Second, our observations relate to the luminous parts of galaxies, which may well have different shapes from the halos. The fraction of mass in gaseous form may have been relevant, particularly in the most recent merger. As gas tends to settle in nearly circular disks, it may influence significantly the shape of the resulting galaxy. More studies of this effect, and detailed simulations of mergers in the protogalactic epoch, may help to clarify these differences.

We thank Ralf Bender, George Djorgovski, Robert Jedrzejewski, Reynier Peletier, and Mike Warren for making their data available in digital form. Discussions with Ed Bertschinger, George Blumenthal, Neil Katz, Peter Quinn, and Paul Schechter are acknowledged. The comments of the referee, James Binney, helped us to improve the paper. M. F. thanks the Harvard Society of Fellows and the Center for Astrophysics for support during the course of this work. G. D. I. appreciates the support of the California Space Institute through grant CS-85-88, and a UCSC Faculty Research grant.

\section{APPENDIX A}

\section{THE PROBABILITY DISTRIBUTIONS}

\section{PROBABILITY DISTRIBUTION $p(\epsilon) d \epsilon$}

We derive an expression for the probability $p(\epsilon) d \epsilon$ of observing an apparent ellipticity $\epsilon$ in the interval $(\epsilon, \epsilon+d \epsilon)$ for random viewing angles and fixed shape. We use the results by F88, who showed that the apparent ellipticity is related to the conical coordinates $\mu, v$ of the vector of projection $p$ by

$$
\epsilon=1-\sqrt{\frac{v(p)}{\mu(p)}} .
$$

See F88 for a more detailed description of the conical coordinates. We introduce the probability distribution $p(\mu, v) d \mu d v$ of finding 
conical coordinates $\mu, v$ in the interval $(\mu, \mu+d \mu),(v, v+d v)$ for arbitrary viewing angles. It is straightforward to derive

$$
p(\mu, v)=\frac{1}{2 \pi} \frac{\mu-v}{\sqrt{-h(\mu) h(v)}},
$$

with

$$
h(\tau)=\left(\tau-a^{2}\right)\left(\tau-b^{2}\right)\left(\tau-c^{2}\right) .
$$

By equation (A1), the probability distribution $p(\mu, \epsilon)$ is given by

$$
d P=p(\epsilon) d \epsilon=\int_{\epsilon(\mu, v)} p(\mu, v) d \mu d v=d \epsilon \int_{\epsilon(\mu, v)} p(\mu, v) \frac{\partial v}{\partial \epsilon} d \mu=d \epsilon \int_{\epsilon(\mu, v)} \frac{(\mu-v) \sqrt{\mu \nu}}{\pi \sqrt{-h(\mu) h(v)}} d \mu,
$$

where $v=(1-\epsilon)^{2} \mu$. We derive

$$
p(\epsilon) d \epsilon= \begin{cases}\frac{(1-e) \sqrt{e}}{\pi} \int_{\mu_{1}}^{\mu_{2}} \frac{\mu^{2}}{\sqrt{-h(\mu) h(e \mu)}} d \mu d \epsilon & \text { for } 0 \leq \epsilon<\epsilon_{1}, \\ 0, & \text { for } \epsilon_{1} \leq \epsilon \leq 1,\end{cases}
$$

where $e=(1-\epsilon)^{2}$. The integration limits $\mu_{1}$ and $\mu_{2}$ depend on the shape of the galaxy. For galaxies with axial ratios $b / a>c / b$, i.e., oblate-triaxial systems, we derive

$$
\left(\mu_{1}, \mu_{2}\right)= \begin{cases}\left(b^{2}, b^{2} / e\right) & \text { for } 0 \leq \epsilon \leq \epsilon_{2} \\ \left(b^{2}, a^{2}\right) & \text { for } \epsilon_{2}<\epsilon \leq \epsilon_{3} \\ \left(c^{2} / e, a^{2}\right) & \text { for } \epsilon_{3}<\epsilon \leq \epsilon_{1}\end{cases}
$$

where $\epsilon_{3}=1-c / b$. For prolate-triaxial galaxies with axial ratios $b / a \leq c / b$ we have

$$
\left(\mu_{1}, \mu_{2}\right)= \begin{cases}\left(b^{2}, b^{2} / e\right) & \text { for } 0 \leq \epsilon \leq \epsilon_{3}, \\ \left(c^{2} / e, b^{2} / e\right) & \text { for } \epsilon_{3}<\epsilon \leq \epsilon_{2}, \\ \left(c^{2} / e, a^{2}\right) & \text { for } \epsilon_{2}<\epsilon \leq \epsilon_{1} .\end{cases}
$$

The integrand in equation (A5) is singular but integrable at these limits. The function $p(\epsilon)$ is generally a hyperelliptic integral, which is easily evaluated numerically. The general characteristics of $p(\epsilon) d \epsilon$ have been described by Binney \& de Vaucouleurs (1981). The present formalism shows that special cases occur not only for oblate and prolate spheroids but also for elliptical disks and for ellipsoids with $b / a=c / a$.

\section{CALCULATION OF $p(\Psi, \epsilon)$ FOR MODELS WITH PERFECT INTRINSICALIGNMENT $\left(\psi_{\text {int }}=0\right)$}

Franx (1988a) showed that the apparent minor axis of a projected ellipsoid is parallel to the tangent of the coordinate line $\mu=\mu(\boldsymbol{p})$ on the unit sphere of projection directions at $\boldsymbol{p}$. It follows that the position angle of the minor axis is given by

$$
\tan ^{2} \Gamma_{\text {minor }}=-\frac{\left(\mu-a^{2}\right)\left(\mu-b^{2}\right)\left(v-c^{2}\right)}{\left(v-a^{2}\right)\left(v-b^{2}\right)\left(\mu-c^{2}\right)} .
$$

Since $\psi_{\text {int }}=0$, for these models $\Psi=\Gamma_{\text {minor }}$. The probability distribution $p(\Psi, \epsilon)$ can be evaluated from

$$
p(\Psi, \epsilon) d \Psi d \epsilon=\sum_{\mu_{i}, v_{i}}\left|\frac{\partial(\mu, v)}{\partial(\Psi, \epsilon)}\right| p(\mu, v) d \Psi d \epsilon,
$$

where $\mu_{i}, v_{i}$ are all the solutions (possibly multiple) of $\mu, v$ for

$$
\Psi(\mu, v)=\Psi, \quad \epsilon(\mu, v)=\epsilon .
$$

The Jacobian in equation (A9) can be written as

$$
\left|\frac{\partial(\mu, v)}{\partial(\Psi, \epsilon)}\right|=4 \mu \sqrt{\mu v}\left[\left(a^{2}-c^{2}\right)\left(b^{2}-c^{2}\right)-\left(\mu-c^{2}\right)\left(v-c^{2}\right)\right] \frac{\sqrt{-h(\mu) h(v)}}{|C(\mu, v)|},
$$

where

$$
\begin{aligned}
C(\mu, v)= & -a^{2}\left(\mu-b^{2}\right)\left(\mu-c^{2}\right)\left(v-b^{2}\right)\left(v-c^{2}\right)-b^{2}\left(\mu-a^{2}\right)\left(\mu-c^{2}\right)\left(v-a^{2}\right)\left(v-c^{2}\right) \\
& +c^{2}\left(\mu-a^{2}\right)\left(\mu-b^{2}\right)\left(v-a^{2}\right)\left(v-b^{2}\right) .
\end{aligned}
$$

The resulting expression for $p(\Psi, \epsilon)$ is

$$
p(\Psi, \epsilon)=\sum_{\mu_{i}, v_{i}}\left[\left(a^{2}-c^{2}\right)\left(b^{2}-c^{2}\right)-\left(\mu-c^{2}\right)\left(v-c^{2}\right)\right] \frac{2 \mu(\mu-v) \sqrt{\mu v}}{\pi|C(\mu, v)|} .
$$


We still have to calculate the solutions of equation (A10). By eliminating $v$ from

$$
\begin{gathered}
e \equiv(1-\epsilon)^{2}=\frac{v}{\mu} \\
\tan ^{2} \Psi \equiv t^{2}=-\frac{\left(\mu-a^{2}\right)\left(\mu-b^{2}\right)\left(v-c^{2}\right)}{\left(v-a^{2}\right)\left(v-b^{2}\right)\left(\mu-c^{2}\right)},
\end{gathered}
$$

we obtain a cubic equation for $\mu$,

$$
a_{3} \mu^{3}+a_{2} \mu^{2}+a_{1} \mu+a_{0}=0
$$

with coefficients

$$
\begin{aligned}
& a_{3}=e+t^{2} e^{2} \\
& a_{2}=-c^{2}\left(1+e^{2} t^{2}\right)-e\left(1+t^{2}\right)\left(a^{2}+b^{2}\right), \\
& a_{1}=c^{2}\left(a^{2}+b^{2}\right)\left(1+e t^{2}\right)+a^{2} b^{2}\left(e+t^{2}\right), \\
& a_{0}=-a^{2} b^{2} c^{2}\left(1+t^{2}\right) .
\end{aligned}
$$

Only those solutions that satisfy

$$
b^{2} \leq \mu \leq a^{2}, \quad c^{2} \leq e \mu \leq b^{2}
$$

are valid. In the general case, equation (A16) has two solutions for all ( $\Psi, \epsilon)$ with $\epsilon<\epsilon_{2}$ and either two or no solutions if $\epsilon_{2}<\epsilon<\epsilon_{1}$, depending on the value of $\Psi$.

The probability distribution $p(\Psi)$ is calculated by integrating $p(\Psi, \mu)$ over $\mu$. The result is

$$
p(\Psi) d \Psi=\frac{1}{\pi \sin ^{2} \Psi} \int_{b^{2}}^{a^{2}} \frac{(\mu-v)}{E(v)} \frac{\left(v-c^{2}\right)}{\left(\mu-c^{2}\right.} d \mu,
$$

where $v=v(\mu, \Psi)$ is the solution for $v$ of $\Psi(v, \mu)=\Psi$ (see eq. [A15]) and

$$
E(v)=\left(v-a^{2}\right)\left(v-b^{2}\right)-\left(v-a^{2}\right)\left(v-c^{2}\right)-\left(v-b^{2}\right)\left(v-c^{2}\right) .
$$

We can write this as an explicit function of $T$ and $\Psi$ :

$$
p(\Psi)=\frac{1}{2 \pi \sin ^{2} \Psi}\left[-\log (1-T)+\int_{0}^{T} \frac{A(s, \Psi, T) d s}{(s+1-T) \sqrt{B(s, \Psi, T)}}\right],
$$

where

$$
\begin{aligned}
& A(s, \Psi, T)=s(s-T)+(s+1-T)(2 s-T) \tan ^{2} \Psi, \\
& B(s, \Psi, T)=s^{2}(s-T)^{2}+2(T-2) s(s-T)(s+1-T) \tan ^{2} \Psi+T^{2}(s+1-T)^{2} \tan ^{2} \Psi .
\end{aligned}
$$

For special values of $T$ and/or $\Psi$ the integral is elementary. For $\Psi=\pi / 2$ we find

$$
p(\pi / 2)=\{1+[(1-T) / T] \log (1-T)\} / \pi .
$$

Oblate models have $T=0$, and $p(\Psi)=\delta(\Psi)$. Prolate models have $T=1$. In this case we obtain

$$
p(\Psi)=-\frac{2 \log (\sin \Psi)}{\pi \cos ^{2} \Psi} .
$$

\section{OBLATE MODELS WITH MISALIGNMENTS $(T=0)$}

Equation (6) in $\S 2.2$ gives the apparent misalignment as a function of the viewing angles and the intrinsic misalignment. Equation (A1) for the apparent ellipticity reduces to

$$
\epsilon=1-\sqrt{\left(1-\epsilon_{1}\right)^{2}+\cos ^{2} \theta\left[1-\left(1-\epsilon_{1}\right)^{2}\right]}
$$

for oblate systems.

It is straightforward to calculate the probability of finding $\epsilon, \Psi$ in the interval $(\epsilon, \epsilon+d \epsilon),(\Psi, \Psi+d \Psi)$

$$
p(\epsilon, \Psi) d \epsilon d \Psi=\left[\sum_{\phi_{i}, \theta_{i}}\left|\frac{\partial(\phi, \theta)}{\partial(\epsilon, \Psi)}\right| p(\phi, \theta)\right] d \epsilon d \Psi=\left[\sum_{\phi_{i}, \theta_{i}}\left|\frac{\partial(\phi, \theta)}{\partial(\epsilon, \Psi)}\right| \frac{\cos \theta}{4 \pi}\right] d \epsilon d \Psi,
$$

where $\phi_{i}, \theta_{i}$ are the solutions of

$$
\epsilon\left(\phi_{i}, \theta_{i}\right)=\epsilon, \quad \Psi\left(\phi_{i}, \theta_{i}\right)=\Psi
$$


No. 1, 1991

We find

$$
p(\epsilon, \Psi)=\frac{1}{4 \pi}\left[\sum_{\phi_{i}, \theta_{i}} \frac{\sin ^{2} \phi}{\sin ^{2} \Psi} \frac{\tan \psi_{\mathrm{int}}}{\left|\sin \theta \cos \phi-\cos \theta \tan \psi_{\mathrm{int}}\right|}\right] \frac{1-\epsilon}{\sqrt{\left[1-\left(1-\epsilon_{1}\right)^{2}\right]\left[(1-\epsilon)^{2}-\left(1-\epsilon_{1}\right)^{2}\right]}} .
$$

The inversion of $\epsilon, \Psi$ to $\phi, \theta$ can be done explicitly. Equation (A26) reduces to

$$
p(\epsilon, \Psi)=\frac{2}{\pi} \frac{C\left(\psi_{\text {int }}, \theta, \Psi\right)}{\pi\left[\cos ^{2} \Psi+\cos ^{2} \theta \sin ^{2} \Psi\right]} \frac{1-\epsilon}{\sqrt{\left[1-\left(1-\epsilon_{1}\right)^{2}\right]\left[(1-\epsilon)^{2}-\left(1-\epsilon_{1}\right)^{2}\right]}},
$$

where

$$
\begin{gathered}
C\left(\psi_{\mathrm{int}}, \theta, \Psi\right)= \begin{cases}\cos \theta & \text { for } \theta<\psi_{\mathrm{int}}, \\
\sin \theta / \sqrt{D\left(\psi_{\mathrm{int}}, \theta, \Psi\right)} & \text { for } \theta>\psi_{\mathrm{int}},\end{cases} \\
D\left(\psi_{\mathrm{int}}, \theta, \Psi\right)=\tan ^{2} \psi_{\mathrm{int}}+\tan ^{2} \psi_{\mathrm{int}} \cos ^{2} \theta \tan ^{2} \Psi-\sin ^{2} \theta \tan ^{2} \Psi, \\
\theta=\arccos \sqrt{\frac{(1-\epsilon)^{2}-\left(1-\epsilon_{1}\right)^{2}}{1-\left(1-\epsilon_{1}\right)^{2}}} .
\end{gathered}
$$

We have left $\theta$ in parts of the expression to avoid unnecessarily long equations. The probability density depends on $\epsilon$ and $\epsilon_{1}$ only through $(1-\epsilon) /\left(1-\epsilon_{1}\right)$, except for a constant scaling factor. Thus the probability densities of all models with constant $\psi_{\text {int }}$ but different $\epsilon_{1}$ are easily transformed into each other.

The integrated probability distribution $p(\Psi)$ is given by

$$
\begin{aligned}
& p(\Psi)=I_{1}+I_{2}, \\
& I_{1}=-\frac{1}{\pi \sin ^{2} \Psi} \log \left(\cos ^{2} \Psi+\cos ^{2} \psi_{\mathrm{int}} \sin ^{2} \Psi\right), \\
& I_{2}=\frac{2}{\pi} \frac{1}{\sqrt{\tan ^{2} \Psi \tan ^{2} \psi_{\mathrm{int}}+\tan ^{2} \Psi}} \int_{c_{1}}^{\cos \psi_{\mathrm{int}}} \frac{\sqrt{1-x^{2}}}{\cos ^{2} \Psi+x^{2} \sin ^{2} \Psi} \frac{d x}{\sqrt{x^{2}+c_{2}}},
\end{aligned}
$$

where

$$
\begin{aligned}
& c_{1}= \begin{cases}0 & \text { if } \quad \Psi \leq \psi_{\mathrm{int}}, \\
1 / \sqrt{-c_{2}} & \text { if } \quad \Psi>\psi_{\mathrm{int}},\end{cases} \\
& c_{2}=\frac{\tan ^{2} \psi_{\mathrm{int}}-\tan ^{2} \Psi}{\tan ^{2} \Psi \tan ^{2} \psi_{\mathrm{int}}+\tan ^{2} \psi_{\mathrm{int}}} .
\end{aligned}
$$

The remaining elliptic integral in $I_{2}$ is evaluated numerically.

\section{APPENDIX B}

\section{AXIAL RATIOS OF PEAKS IN LINEAR GAUSSIAN RANDOM FIELDS}

BBKS derive the distribution of shapes in terms of the parameters $e$ and $p$, which are related to the axis lengths of a triaxial ellipsoid by

$$
e=\frac{c^{-2}-a^{-2}}{2\left(a^{-2}+b^{-2}+c^{-2}\right)}, \quad p=\frac{a^{-2}-2 b^{-2}+c^{-2}}{2\left(a^{-2}+b^{-2}+c^{-2}\right)}
$$

These are related to our parameters $\epsilon_{1}$ and $T$ by

$$
e_{1}=\left(1-\epsilon_{1}\right)^{2}=\frac{1+p-3 e}{1+p+3 e}, \quad T=\frac{(p-e)(1+p+3 e)}{2(2 p-1) e}
$$

so that

$$
e=\frac{\left(1-e_{1}^{2}\right)\left[1-T\left(1-e_{1}^{2}\right)\right]}{2\left\{\left(e_{1}^{2}+1\right)\left[1-T\left(1-e_{1}^{2}\right)\right]+e_{1}^{2}\right\}}, \quad p=\frac{\left(1+e_{1}^{2}\right)\left[1-T\left(1-e_{1}^{2}\right)\right]-2 e_{1}^{2}}{2\left\{\left(e_{1}^{2}+1\right)\left[1-T\left(1-e_{1}^{2}\right)\right]+e_{1}^{2}\right\}} .
$$

BBKS proved that the probability distribution of $e, p$ depends only on the parameter $x$, which is a measure of the size of the peak:

$$
p(e, p \mid x)=\frac{3^{2} 5^{5 / 2}}{\sqrt{2 \pi}} \frac{x^{8}}{f(x)} \exp \left[-\left(\frac{5}{2}\right) x^{2}\left(3 e^{2}+p^{2}\right)\right] W(e, p) \text {. }
$$


The function $f(x)$ is given by BBKS in equation (A15), and the polynomial $W(e, p)$ in equation (C4). It is straightforward to derive for the probability distribution of $\epsilon_{1}, T$, for given $x$

$$
p\left(\epsilon_{1}, T \mid x\right)=\frac{5^{5 / 2} 6}{\sqrt{2 \pi}} \frac{x^{8}}{f(x)} \exp \left[-\left(\frac{5}{2}\right) x^{2}\left(3 e^{2}+p^{2}\right)\right] e_{1} e^{2}(1-2 p)^{3}\left(e^{2}-p^{2}\right)(1+p+3 e)^{2} .
$$

\section{REFERENCES}

Bardeen, J. M., Bond, J. R., Kaiser, N., \& Szalay, A. S. 1986, ApJ, 304, 15 (BBKS)

Barnes, J., \& Efstathiou, G. 1987, ApJ, 319, 575

Bender, R. 1988, A\&A, 202, L5

. 1990, A\&A, 229, 441

Bender, R., Döbereiner, S., \& Möllenhof, C. 1988, A\&AS, 74, 385

Bender, R., \& Möllenhof, C. 1987, A\&A, 177, 71

Bender, R., \& Nieto, J. L. 1990, A\&A, 239, 97

Bertola, F., Bettoni, D., Danziger, J., Sadler, E., Sparke, L. S., \& de Zeeuw, P. T. 1991, ApJ, 373, 369

Binggeli, B. 1980, A\&A, 82, 289

Binney, J. J. 1978, MNRAS, 183, 779

. 1985, MNRAS, 212, 767

Binney, J. J., \& de Vaucouleurs, G. 1981, MNRAS, 194, 679

Binney, J. J., \& Tremaine, S. D. 1987, Galactic Dynamics (Princeton: Princeton Univ. Press)

Blumenthal, G. R., Faber, S. M., Primack, J. R., \& Rees, M. J. 1984, Nature, 311,517

Caldwell, N. 1984, ApJ, 278, 96

Caldwell, N., Kirshner, R. P., \& Richstone, D. O. 1986, ApJ, 305, 136

Capaccioli, M. 1987, in IAU Symposium 127, Structure and Dynamics of Elliptical Galaxies, ed. P. T. de Zeeuw (Dordrecht: Reidel), 47

Carter, D. 1987, ApJ, 312, 514

Contopoulos, G. 1956, Zs. Ap., 39, 126

Davies, R. L., \& Birkinshaw, M. 1986, ApJ, 303, L45

- 1988, ApJS, 68, 409 (DB)

Davies, R. L., Efstathiou, G., Falls, S. M., Illingworth, G., \& Schechter, P. L. 1983, ApJ, 266, 41

Davies, R. L., \& Illingworth, G. D. 1986, ApJ, 302, 234

de Vaucouleurs, G., de Vaucouleurs, A., \& Corwin, H. G. 1976, Second Reference Catalogue of Bright Galaxies (Univ. Texas Monographs in Astronomy,

No. 2; Austin: Univ. Texas Press) (RC2)

de Zeeuw, P. T., \& Franx, M. 1989, ApJ, 343, 617 1991, ARA\&A, 29, 239

Djorgovski, S. G. 1985, Ph.D. thesis, Univ. California, Berkeley

Doroshkevich, A. G. 1970, Astrophysica, 6, 320

Dubinski, J., \& Carlberg, R. G. 1991, ApJ, 378, 496

Faber, S. M., Wegner, G., Burstein, D., Davies, R. L., Dressler, A., Lynden-Bell, D., \& Terlevich, R. J. 1989, ApJS, 69, 763 (7SAM)

Fall, S. M., \& Frenk, C. S. 1983, AJ, 88, 1626
Fasano, G., \& Vio, R. 1991, MNRAS, 249, 629

Franx, M. 1988a, MNRAS, 231, 285 (F88)

. 1988b, Ph.D. thesis, Leiden Univ.

Franx, M., \& Illingworth, G. D. 1988, ApJ, 327, L55

Franx, M., Illingworth, G. D., \& Heckman, T. M. 1989a, AJ, 98, 538 1989b, ApJ, 344, 613 (FIH)

Frenk, C. S., White, S. D. M., Davis, M., \& Efstathiou, G. 1988, ApJ, 327, 507

Heckman, T. M., Smith, E. P., Baum, S. A., van Breugel, W. J. M., Miley, G. K., Illingworth, G. D., Bothun, G. D., \& Balick, B. 1986, ApJ, 311, 526

Heiligman, G. M., \& Schwarzschild, M. 1979, ApJ, 258, 490

Illingworth, G. D., \& Franx, M. 1990, in ASP Conf. Ser. 10, Evolution of the

Universe of Galaxies, ed. R. G. Kron (San Francisco: ASP), 82

Jedrzejewski, R. I. 1987, MNRAS, 226, 747

Jedrzejewski, R. I., \& Schechter, P. L. 1989, AJ, 98, 147 (JS)

Katz, N., \& Gunn, J. E. 1991, ApJ, 377, 365

Levison, H. F., \& Richstone, D. O. 1987, ApJ, 314, 476

Lucy, L. B. 1974, AJ, 79, 745

Merritt, D. 1982, AJ, 87, 1279

Miralda-Escudé, J., \& Schwarzschild, M. 1989, ApJ, 339, 752

Peebles, P. J. E. 1982, ApJ, 263, L1

Peletier, R. F., Illingworth, G. D., Davies, R. L., Davies, L. E., \& Cawson, M. C. 1990, AJ, 100, 1091

Porter, A. C., Schneider, D. P., \& Hoessel, J. G. 1991, AJ, 101, 1561

Press, W. H., Flannery, B. P., Teukolsky, S. A., \& Vetterling, W. T. 1986, Numerical Recipes (Cambridge: Cambridge Univ. Press)

Quinn, P. J., \& Zurek, W. H. 1988, ApJ, 331, 1

Rix, H. W., \& White, S. D. M. 1990, ApJ, 362, 52

Sadler, E. M., \& Gerhard, O. E. 1985, MNRAS, 214, 177

Schechter, P. L. 1987, in IAU Symposium 127, Structure and Dynamics of Elliptical Galaxies, ed. P. T. de Zeeuw (Dordrecht: Reidel), 217

Schwarzschild, M. 1979, ApJ, 232, 236

Stark, A. A. 1977, ApJ, 213, 368

Statler, T. S. 1987, ApJ, 321, 113

Strom, K. M., \& Strom, S. E. 1978, AJ, 83, 1239

Wagner, S. J., Bender, R., \& Möllenhof, C. 1988, A\&A, 195, L5

Warren, M. S., Zurek, W. H., Quinn, P. J., \& Salmon, J. K. 1991a, preprint .1991b, ApJ, submitted

White, S. D. M., \& Ostriker, J. P. 1990, ApJ, 349, 22

Wilkinson, A., Sharples, R. M., Fosbury, R. A. E., \& Wallace, P. T. 1986, MNRAS, 218, 297 